\section{Government finance}

\section{Public sector}

\section{(Tables 18.1 to 18.3 and 18.5 )}

In Table 18.1 the term public sector describes the consolidation of central government, local government and public corporations. General government is the consolidated total of central government and local government. The table shows details of the key public sector finances' indicators, consistent with the European System of Accounts 1995 (ESA95), by sub-sector.

The concepts in Table 18.1 are consistent with the format for public finances in the Economic and Fiscal Strategy Report (EFSR), published by HM Treasury on 11 June 1998, and the Budget. The public sector current budget is equivalent to net saving in national accounts plus capital tax receipts. Net investment is gross capital formation, plus payments less receipts of investment grants, less depreciation. Net borrowing is net investment less current budget. Net borrowing differs from the net cash requirement (see below) in that it is measured on an accruals basis whereas the net cash requirement is mainly a cash measure which includes some financial transactions. Table 18.2 shows the public sector key fiscal balances. The table shows the component detail of the public sector key fiscal balance by economic category. The tables are consistent with the Budget.

Table 18.3 shows public sector net debt. Public sector net debt consists of the public sector's financial liabilities at face value, minus its liquid assets - mainly foreign currency exchange reserves and bank deposits. General government gross debt (consolidated) in Table 18.3 is consistent with the definition of general government gross debt reported to the European Commission under the requirements of the Maastricht Treaty.

More information on the concepts in Table 18.1, 18.2 and 18.3 can be found in a guide to monthly public sector finance statistics, GSS Methodology Series No 12, the ONS Statistical Bulletin Public Sector Finances and Financial Statistics Explanatory Handbook.

Table 18.6 shows the taxes and National Insurance contributions paid to central government, local government, and to the institutions of the European Union. The table is the same as Table 11.1 of the National Accounts Blue Book. More information on the data and concepts in the table can be found in Chapter 11 of the Blue Book.

\section{Consolidated Fund and National Loans Fund}

\section{(Tables 18.4, 18.5 and 18.7)}

The central government embraces all bodies for whose activities a Minister of the Crown, or other responsible person, is accountable to Parliament. It includes, in addition to the ordinary government departments, a number of bodies administering public policy, but without the substantial degree of financial independence which characterises the public corporations. It also includes certain extra-budgetary funds and accounts controlled by departments.

The government's financial transactions are handled through a number of statutory funds or accounts. The most important of these is the Consolidated Fund, which is the government's main account with the Bank of England. Up to 31 March 1968 the Consolidated Fund was virtually synonymous with the term 'Exchequer', which was then the government's central cash account. From 1 April 1968 the National Loans Fund, with a separate account at the Bank of England, was set up by the National Loans Act 1968. The general effect of this Act was to remove from the Consolidated Fund most of the government's domestic lending and the whole of the government's borrowing transactions, and to provide for them to be brought to account in the National Loans Fund.

Revenue from taxation and miscellaneous receipts, including interest and dividends on loans made from votes, continue to be paid into the Consolidated Fund.

After meeting the ordinary expenditure on Supply Services and the Consolidated Fund Standing Services, the surplus or deficit of the Consolidated Fund (Table 18.4), is payable into or met by the National Loans Fund. Table 18.4 also provides a summary of the transactions of the National Loans Fund. The service of the National Debt, previously borne by the Consolidated Fund, is now met from the National Loans Fund which receives:

(a) interest payable on loans to the nationalised industries, local authorities and other bodies, whether the loans were made before or after 1 April 1968, and

(b) the profits of the Issue Department of the Bank of England, mainly derived from interest on government securities, which were formerly paid into the Exchange Equalisation Account. The net cost of servicing the National Debt after applying these interest receipts and similar items is a charge on the Consolidated Fund as part of the standing services. Details of National Loans Fund loans outstanding are shown in Table 18.5. Details of borrowing and repayments of debt, other than loans from the National Loans Fund, are shown in Table 18.7. 


\section{Income tax}

\section{(Table 18.11, 18. 12)}

Following the introduction of Independent Taxation from 1990/91, the Married Couple's Allowance was introduced. It is payable in addition to the Personal Allowance and between 1990/91 and 1992/93 went to the husband unless the transfer condition was met. The condition was that the husband was unable to make full use of the allowance himself and in that case he could transfer part or all of the Married Couple's Allowance to his wife. In 1993/94 all or half of the allowance could be transferred to the wife if the couple had agreed beforehand. The wife has the right to claim half the allowance. The Married Couple's Allowance, and allowances linked to it, were restricted to 20 per cent in 1994/95 and to 15 per cent from 1995/96. From 2000/01 only people born before 6 April 1935 are entitled to Married Couple's Allowance.

The age allowance replaces the single allowance, provided the taxpayer's income is below the limits shown in the table. From 1989/90, for incomes in excess of the limits, the allowance is reduced by $£ 1$ for each additional $f 2$ of income until the ordinary limit is reached (before it was $\mathrm{f} 2$ for each $\mathrm{f} 3$ of additional income). The relief is due where the taxpayer is aged 65 and over in the year of assessment.

The additional Personal Allowance could be claimed by a single parent (or by a married man if his wife was totally incapacitated) who maintained a resident child at his or her own expense. Widow's Bereavement Allowance was due to a widow in the year of her husband's death and in the following year provided the widow had not remarried before the beginning of that year. Both the additional Personal Allowance and the Widow's Bereavement Allowance were abolished from April 2000.

The Blind Person's Allowance may be claimed by blind persons (in England and Wales, registered as blind by a local authority) and surplus Blind Person's Allowance may be transferred to a husband or wife. Relief on life assurance premiums is given by deduction from the premium payable. From 1984/85 it is confined to policies taken out before 14 March 1984.

From 1993/94 until 1998/99 a number of taxpayers with taxable income in excess of the lower rate limit only paid tax at the lower rate. This was because it was only their dividend income and (from 1996/97) their savings income which took their taxable income above the lower rate limit but below the basic rate limit, and such income was chargeable to tax at the lower rate and not the basic rate.
In 1999/2000 the 10 per cent starting rate replaced the lower rate and taxpayers with savings or dividend income at the basic rate of tax are taxed at 20 per cent and 10 per cent respectively. Before 1999/2000 these people would have been classified as lower rate taxpayers. The 10 per cent starting rate was abolished in 2009/10.

\section{Rateable values}

(Table 18.14)

Major changes to local government finance in England and Wales took effect from 1 April 1990. These included the abolition of domestic rating (replaced by the Community Charge, then replaced in 1993 by the Council Tax), the revaluation of all non-domestic properties, and the introduction of the Uniform Business Rate. Also in 1990, a new classification scheme was introduced which has resulted in differences in coverage. Further differences are caused by legislative changes which have changed the treatment of certain types of property. There was little change in the total rateable value of non-domestic properties when all these properties were revalued in April 1995. Rateable values for offices fell and there was a rise for all other property types shown in the table.

With effect from 1 April 2000, all non-domestic properties were revalued. Overall there was an increase in rateable values of over 25 per cent compared to the last year of the 1995 list. The largest proportionate increase was for offices and cinemas, with all property types given in the table showing rises.

The latest revaluation affecting all non-domestic properties took effect from 1 April 2005. In this revaluation the overall increase in rateable values between 1 April of the first year of the new list and the same day on the last year of the 2000 list was 17 per cent. The largest proportionate increase was for theatres and music halls with again all property types in the table showing rises.

\section{Local authority capital expenditure and receipts}

\section{(Table 18.17)}

Authorities finance capital spending in a number of ways, including use of their own revenue funds, borrowing or grants, and contributions from elsewhere. Until 31 March 2004, the capital finance system laid down in Part 4 of the Local Government and Housing Act 1989 (the '1989 Act') provided the framework within which authorities were permitted to finance capital spending from sources other than revenue - that is by the use of borrowing, long-term credit or capital receipts. 
Until 31 March 2004, capital spending could be financed by:

- revenue resources - either the General Fund Revenue Account, the Housing Revenue Account (HRA) or the Major Repairs Reserve - but an authority could not charge council tenants for spending on general services, or spending on council houses to local taxpayers

- borrowing or long-term credit as authorised by the credit approvals issued by central government. Credit approvals were normally accompanied by an element of Revenue Support Grant (RSG) covering most of the costs of borrowing

- grants received from central government

- contributions or grants from elsewhere - including the National Lottery; NDPBs such as Sport England, English Heritage and Natural England; private sector partners; capital receipts (that is, proceeds from the sale of land, buildings or other fixed assets); and sums set aside as Provision for Credit Liabilities (PCL). This required the use of a credit approval, unless the authority was debt-free

From 1 April 2004, capital spending can be financed in the same ways, except that central government no longer issues credit approvals to allow authorities to finance capital spending by borrowing. However, it continues to provide financial support in the usual way, via RSG or HRA subsidy, towards some capital spending financed by borrowing that is Supported Capital Expenditure (Revenue). Authorities are now free to finance capital spending by self-financed borrowing within limits of affordability set, having regard to the 2003 Act and the CIPFA Prudential Code. The concept of PCL has not been carried forward into the new system, although authorities which were debt-free and had a negative credit ceiling at the end of the old system could still spend amounts of PCL built up under the old rules.

In 2008/09 capital receipts fell to $£ 1.4$ billion, a year-on-year decrease of 66 per cent. This fall reflects the effect of the economic climate over that period on local authority sales of assets.

In 2008/09 capital expenditure of almost $£ 4.2$ billion (about 21 per cent) was financed by self-financed borrowing, an increase of 33 per cent from the amount financed in 2007/08.

In 2008/09, government grants accounted for 28 per cent of the total financing. Financing by government grant in 2007/08 was affected by the grant of $f 1.7$ billion paid by the Department for Transport to the Greater London Authority (GLA) in respect of Metronet liabilities; this caused government grants to account for 34 per cent of the total financing for 2007/08.

\section{Local authority financing for capital expenditure}

\section{(Table 18.18)}

Capital spending by local authorities is mainly for buying, constructing or improving physical assets such as:

- buildings - schools, houses, libraries and museums, police and fire stations

- land - for development, roads, playing fields

- vehicles, plant and machinery - including street lighting and road signs

It also includes grants and advances made to the private sector or the rest of the public sector for capital purposes, such as advances to Registered Social Landlords. Local authority capital expenditure more than doubled between 2001/02 and 2007/08.

The underlying trend in capital expenditure (excluding an exceptional event in 2007/08) shows an increase of 9 per cent from 2007/08 to 2008/09. The exceptional event was the payment by the Greater London Authority (Transport for London) of $\mathrm{f} 1.7$ billion to Metronet in 2007/08.

New construction, conversion and renovation forms the major part of capital spending. The largest increases in capital expenditure in 2008/09 were in police (44 per cent), and education (22 per cent). Capital expenditure on transport increased by 14 per cent, allowing for the Greater London Authority's grant payment via TfL in respect of Metronet in 2007/08. Between 2004/05 and 2008/09 capital expenditure on transport has risen from 20 per cent to 24 per cent of the total, while capital expenditure on housing has fallen from 28 per cent to 25 per cent of the total. 


\begin{tabular}{|c|c|c|c|c|c|c|c|c|c|c|c|c|}
\hline & & $\begin{array}{r}1999 \\
100\end{array}$ & $\begin{array}{r}2000 \\
101\end{array}$ & $\begin{array}{r}2001 \\
102\end{array}$ & $\begin{array}{r}2002 \\
103\end{array}$ & $\begin{array}{r}2003 \\
104\end{array}$ & $\begin{array}{r}2004 \\
105\end{array}$ & $\begin{array}{r}2005 \\
106\end{array}$ & $\begin{array}{r}2006 \\
107\end{array}$ & $\begin{array}{r}2007 \\
108\end{array}$ & $\begin{array}{r}2008 \\
109\end{array}$ & $\begin{array}{r}2009 \\
/ 10\end{array}$ \\
\hline \multicolumn{13}{|l|}{ Surplus on current budget ${ }^{3}$} \\
\hline Central Government & ANLV & 24401 & 26756 & 13812 & -8121 & -17559 & -17757 & -13495 & -5795 & -5824 & -47675 & .. \\
\hline Local government & NMMX & -4507 & -3790 & -3909 & -4960 & -3245 & -3135 & -5094 & -3016 & -2849 & -4363 & .. \\
\hline General Government & ANLW & 20878 & 21996 & 10861 & -11103 & -19005 & & -18657 & -8292 & -12103 & -53658 & .. \\
\hline Public corporations & IL6M & 983 & 808 & 1311 & 541 & 1694 & 2003 & 4573 & 3685 & 3640 & 2494 & .. \\
\hline Public sector & ANMU & 20995 & 23432 & 12144 & -11323 & -17418 & -19249 & -13943 & -4919 & -4846 & -49638 & .. \\
\hline \multicolumn{13}{|l|}{ Net investment ${ }^{4}$} \\
\hline Central government & -ANNS & 9493 & 8947 & 14234 & 18231 & 19570 & 20798 & 20000 & 26834 & 32882 & 44106 & .. \\
\hline Local government & -ANNT & -832 & -1882 & -1824 & -3595 & -682 & 1756 & 419 & -121 & -3779 & 1250 & .. \\
\hline General Government & -ANNV & 6461 & 6574 & 10951 & 12487 & 16371 & & 19721 & 25955 & 29980 & 46916 & .. \\
\hline Public corporations & -JSH6 & -2737 & -2180 & -1435 & -1761 & -2493 & -653 & 3163 & -645 & -1035 & -7459 & .. \\
\hline Public sector & - ANNW & 5501 & 5125 & 11901 & 13805 & 15623 & 20574 & 23466 & 25917 & 29176 & 36664 & .. \\
\hline \multicolumn{13}{|l|}{ Net borrowing ${ }^{5}$} \\
\hline Central government & -NMFJ & -14908 & -17809 & 422 & 26352 & 37129 & 38555 & 33495 & 32629 & 38706 & 91781 & .. \\
\hline Local government & $-\mathrm{NMOE}$ & 3134 & 2490 & 2081 & 1078 & 99 & 3924 & 5324 & 2537 & -9 & 4474 & .. \\
\hline Public sector & $-A N N X$ & -15494 & -18307 & -243 & 25128 & 33041 & 39823 & 37409 & 30836 & 34022 & 86302 & .. \\
\hline \multicolumn{13}{|l|}{ Net cash requirement } \\
\hline Central government ${ }^{6}$ & RUUX & -10664 & -37251 & 3366 & 24214 & 42717 & 37454 & 35908 & 36891 & 29621 & 162513 & .. \\
\hline Local government & ABEG & 979 & -611 & -423 & -2715 & -2712 & 1270 & 4153 & 58 & -723 & 4401 & .. \\
\hline General Government & RUUS & -9685 & -37862 & 2943 & 21499 & 40005 & 38724 & 40061 & 36949 & 28898 & 166914 & .. \\
\hline Public corporations & IL6F & 1622 & 1324 & 1135 & 3063 & -1557 & -303 & 335 & -1839 & -7377 & -107358 & .. \\
\hline Public sector & RURQ & -8063 & -36538 & 4078 & 24562 & 38448 & 38421 & 40396 & 35110 & 21521 & 59556 & .. \\
\hline \multicolumn{13}{|l|}{ Public sector debt } \\
\hline Public sector net debt & BKQK & 344352 & 311143 & 314257 & 346034 & 381502 & 422065 & 461671 & 497806 & 621588 & 742317 & 889988 \\
\hline $\begin{array}{l}\text { Public sector net debt ( } £ \text { billion) } \\
\text { Public sector net debt as }\end{array}$ & RUTN & 344.4 & 311.1 & 314.3 & 346.0 & 381.5 & 422.1 & 461.7 & 497.8 & 621.6 & 742.8 & \\
\hline $\begin{array}{l}\text { a percentage of GDP } \\
\text { Excluding financial interventions }\end{array}$ & RUTO & 35.6 & 30.7 & 29.7 & 30.8 & 32.1 & 34.0 & 35.3 & 36.0 & 43.0 & 52.9 & 62.0 \\
\hline Net debt & $\mathrm{HF} 6 \mathrm{~W}$ & 344.4 & 311.1 & 314.3 & 346.0 & 381.5 & 422.1 & 461.7 & 497.8 & 527.2 & 617.1 & .. \\
\hline Net debt as a \% GDP & $\mathrm{HF} 6 \mathrm{X}$ & 35.6 & 30.7 & 29.7 & 30.8 & 32.1 & 34.0 & 35.3 & 36.0 & 36.5 & 43.8 & \\
\hline
\end{tabular}

1 National accounts entities as defined under the European System of Ac- 5 Net investment less surplus on current budget. A version of General governcounts 1995 (ESA95) consistent with the latest national accounts. See ment net borrowing is reported to the European Commision under the requirechapter text. ments of the Maastricht Treaty.

2 Unless otherwise stated.

6 Central government net cash requirement (own account).

3 Net saving plus capital taxes.

4 Gross capital formation plus payments less receipts of investment grants Source: Office for National Statistics: 02070142124 less depreciation. 


\begin{tabular}{|c|c|c|c|c|c|c|c|c|c|c|c|}
\hline & $\begin{array}{r}1998 \\
/ 99\end{array}$ & $\begin{array}{r}1999 \\
/ 00\end{array}$ & $\begin{array}{r}2000 \\
/ 01\end{array}$ & $\begin{array}{r}2001 \\
/ 02\end{array}$ & $\begin{array}{r}2002 \\
103\end{array}$ & $\begin{array}{r}2003 \\
/ 04\end{array}$ & $\begin{array}{r}2004 \\
/ 05\end{array}$ & $\begin{array}{r}2005 \\
106\end{array}$ & $\begin{array}{r}2006 \\
107\end{array}$ & $\begin{array}{r}2007 \\
/ 08\end{array}$ & $\begin{array}{r}2008 \\
109\end{array}$ \\
\hline
\end{tabular}

Current receipts

Taxes on income and wealth

Taxes on production

Other current taxes ${ }^{2}$

Taxes on capital

Social contributions

Gross operating surplus

Interest and dividends from private sector and Rest of World

Rent and other current transfers ${ }^{3}$

\section{Total current receipts}

\section{Current expenditure}

Current expenditure on goods and services ${ }^{4}$

Subsidies

Social benefits

Net current grants abroad

Other current grants

Interest and dividends paid to private sector and Rest of World

\section{Total current expenditure}

Saving, gross plus capital taxes

Depreciation

Surplus on current budget

Net investment

Gross fixed capital formation ${ }^{6}$

Less depreciation

Increase in inventories and valuables

Capital grants to private sector and Rest of World

Capital grants from private sector and Rest of World

Total net investment

Net borrowing ${ }^{7}$

Financial transactions determining net cash requirement

Net lending to private sector and Rest of World

Net acquisition of UK company securities

Accounts receivable/payable

Adjustment for interest on gilts

Other financial transcations ${ }^{8}$

Public sector net cash requirement $\begin{array}{llllllllllllllllll} & \end{array}$

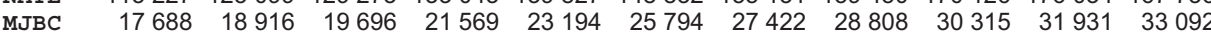

$\begin{array}{llrrrrrrrrrr}\text { NMGI } & 1804 & 2054 & 2236 & 2383 & 2370 & 2521 & 2941 & 3276 & 3618 & 3890 & 23783\end{array}$

$\begin{array}{llllllllllllll}\text { ANBO } & 54746 & 56935 & 62068 & 63162 & 63529 & 75148 & 80923 & 85559 & 90818 & 95234 & 96951\end{array}$

$\begin{array}{lllllllllllll}\text { ANBP } & 16822 & 16949 & 16669 & 16907 & 17106 & 18393 & 18611 & 21574 & 22647 & 22912 & 22649\end{array}$

$\begin{array}{llllllllllll}\text { ANBQ } & 5283 & 4368 & 6226 & 4898 & 4606 & 4662 & 6079 & 6729 & 6230 & 8242 & 6623\end{array}$

$\begin{array}{lllllllllllll}\text { ANBS } & 891 & 1037 & 2036 & 2427 & 2470 & 2036 & 1964 & 1969 & 1864 & 1762 & 1761\end{array}$

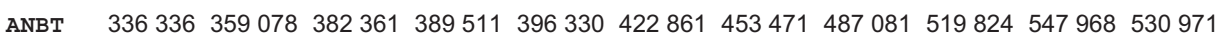

GZSN 159443172299185875198935217512236606255961273737287767300117315724 $\begin{array}{llllllllllll}\text { NMRL } & 4164 & 4215 & 4412 & 4504 & 6043 & 6787 & 7461 & 8140 & 8843 & 9565 & 8589\end{array}$ $\begin{array}{llllllllllllll}\text { ANLY } & 106585 & 105555 & 108010 & 118269 & 122636 & 130799 & 136848 & 142370 & 147429 & 157821 & 171833\end{array}$

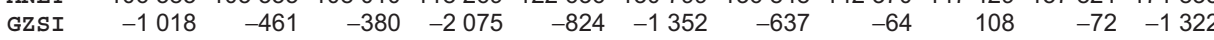
$\begin{array}{llllllllllllll}\text { NNAI } & 15199 & 19106 & 21676 & 23932 & 27555 & 30369 & 32502 & 34079 & 34886 & 37110 & 37089\end{array}$ $\begin{array}{lllllllllllll}\text { ANLO } & 29289 & 25297 & 26400 & 22495 & 21453 & 22822 & 24955 & 26816 & 28800 & 31389 & 31570\end{array}$ ANLT $\overline{313662} \overline{326011} \overline{345993} \overline{366060} \overline{394375} \overline{426010} \overline{457029} \overline{485014} \overline{507765} \overline{535803} \overline{563711}$

$\begin{array}{lllllllllllll}\text { ANSP } & 22674 & 33067 & 36368 & 23451 & 1955 & -3149 & -3558 & 2067 & 12059 & 12165 & -32740\end{array}$

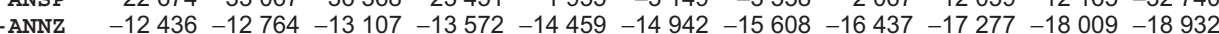

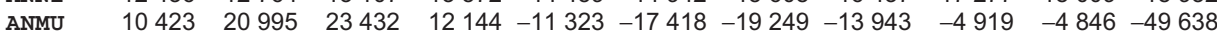

$\begin{array}{llllllllllllll}\text { ANSQ } & 14061 & 14150 & 13283 & 17308 & 20125 & 21079 & 25644 & 27857 & 28971 & 32726 & 41438\end{array}$

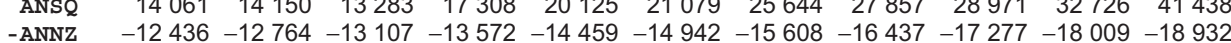

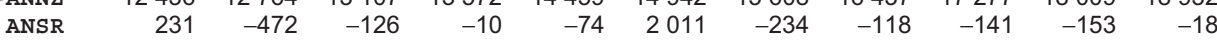
$\begin{array}{lllllllllllll}\text { ANSS } & 4942 & 4371 & 3875 & 7958 & 7564 & 10142 & 11046 & 12451 & 15335 & 14776 & 41300\end{array}$ $\begin{array}{lllllllllllll}- \text { ANST } & -367 & -427 & -756 & -989 & -1091 & -1352 & -972 & -1202 & -1413 & -1089 & -26 & 900\end{array}$ -ANNW $\overline{5955} \overline{5501} \overline{5125} \overline{11901} \overline{13805} \overline{15623} \overline{20574} \overline{23466} \overline{25917} \overline{29176} \overline{36664}$ $\begin{array}{lllllllllllllll}- \text { ANNX } & -4468 & -15494 & -18307 & -243 & 25128 & 33041 & 39823 & 37409 & 30836 & 34022 & 86302\end{array}$

\begin{tabular}{|c|c|c|c|c|c|c|c|c|c|c|c|}
\hline ANSU & 171 & 2212 & 3174 & 2674 & 2736 & 2641 & 925 & 874 & 435 & 4214 & 5452 \\
\hline ANSV & 704 & -310 & 949 & -394 & 765 & 355 & 521 & 655 & -2270 & -2104 & \\
\hline SW & 803 & 8393 & -17163 & 2210 & -2779 & 9031 & 2453 & 2370 & 9105 & -8988 & \\
\hline $\mathrm{sx}$ & -2446 & -1294 & -2630 & -361 & -1444 & -1187 & -2304 & -2749 & -1279 & -4619 & \\
\hline SY & -909 & -1570 & -2561 & 192 & 156 & -5433 & -2997 & 1837 & -1717 & -1004 & -57 \\
\hline & -6145 & -8063 & -36538 & 4078 & 24562 & 38448 & 38421 & 40396 & 35110 & 215 & \\
\hline
\end{tabular}

1 See chapter text.

5 Net of current grants received from abroad.

2 Includes domestic rates, council tax, community charge, motor vehicle duty 6 Including net acquisition of land.

paid by household and some licence fees.

7 Net investment less surplus on current budget.

3 ESA95 transactions D44, D45, D74, D75 and D72-D71: includes rent of 8 Includes statistical discrepancy, finance leasing and similar borrowing, insurland, oil royalties, other property income and fines.

4 Includes non-trading capital consumption. 
$£$ million

\begin{tabular}{rrrrrrrrrr}
\hline & 2001 & 2002 & 2003 & 2004 & 2005 & 2006 & 2007 & 2008 & 2009 \\
109 & 102 & 103 & 104 & 105 & 106 & 107 & 108 & 109
\end{tabular}

Central government sterling gross debt:

British government stock Conventional gilts Index linked gilts

Total

Sterling Treasury bills

National savings

Tax instruments

Other sterling debt ${ }^{2}$

Central government sterling gross debt total

Central government foreign currency gross debt:

US\$ bonds

ECU bonds

ECU/Euro Treasury notes

Other foreign currency debt

Central government foreign currency gross debt total

Central government gross debt total

Local government gross debt total

less

Central government holdings of local government debt

Local government holdings of central government debt

General government gross debt (consolidated)

Public corporations gross debt

BKPK

BKPI

BKPM

BKPJ

ACUA

ACRV

BKSK

BKSL

$200833206119 \quad 232877 \quad 261373 \quad 287481 \quad 306489 \quad 320622 \quad 426107$

$\begin{array}{lllllllll}70417 & 75966 & 78982 & 86749 & 98654 & 113090 & 132404 & 154038\end{array}$

$271250 \quad 282085 \quad 311859 \quad 348122 \quad 386135 \quad 419579 \quad 453026 \quad 580145$

$\begin{array}{llllllll}9700 & 15000 & 19300 & 20350 & 19100 & 15600 & 17569 & 43748\end{array}$

$\begin{array}{llllllll}62275 & 63087 & 66522 & 68504 & 73365 & 78885 & 84768 & 97202\end{array}$

$\begin{array}{rrrrrrrr}478 & 376 & 407 & 350 & 308 & 353 & 428 & 1121\end{array}$

$\begin{array}{llllllll}28276 & 32711 & 35032 & 32279 & 36481 & 41261 & 39373 & 57702\end{array}$

$\begin{array}{llllllllll}371979 & 393259 & 433120 & 469605 & 515389 & 555678 & 595164 & 779 & 918 & 989122\end{array}$

608511

178170

786681

62866

98719

819

40037

BKPG

FYSJ

EYSV

BKPH

BKPI

BKPW

EYKP

-EYKZ

-EYLA

BKPX

EYYD

Central government holdings of public corporations debt -EYXY

Local government holdings of public corporations debt -EYXZ

Public corporations holdings of central government debt -BKPZ

Public corporations holdings of local government debt

- EYXV

Public sector gross debt (consolidated)

BKQA

$$
\begin{array}{rrrrrrr}
2107 & - & 1632 & 1587 & 1730 & 1530 & 1509 \\
- & - & - & - & - & - & - \\
1225 & - & - & - & - & - & - \\
243 & 172 & 105 & 57 & 1 & - & - \\
3575 & 172 & 1738 & 1644 & 1731 & 1530 & 1509
\end{array}
$$

$375554 \quad 393431 \quad 434858 \quad 471249 \quad 517120 \quad 557208 \quad 596673 \quad 779918$

$\begin{array}{lllllllll}52566 & 51353 & 50547 & 53300 & 60114 & 62425 & 66371 & 67301\end{array}$

989122

$17530+-448$

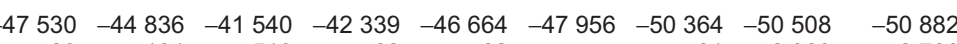

$\begin{array}{lllllllll}-29 & -184 & -510 & -62 & -62 & - & -81 & -2960 & -2706\end{array}$

$380561399764 \quad 443355 \quad 482148 \quad 530508 \quad 571677 \quad 612599 \quad 793751 \quad 1003685$

$4308-417$

$\begin{array}{llllllll}-4171 & -5188 & -5740 & -5631 & -4984 & -5092 & -4879 & -5617\end{array}$

$\begin{array}{lllllllll}638 & -4928 & -4780 & -5080 & -2822 & -2255 & -4119 & -3947 & -3292\end{array}$

Public sector liquid assets:

Official reserves

Central government deposits ${ }^{3}$

Other central government

Local government deposits ${ }^{3}$

Other local government short term assets

Public corporations deposits ${ }^{3}$

Other public corporations short term assets

Public sector liquid assets total

$\begin{array}{lllllllll}\text { AIPD } & 28055 & 26387 & 25266 & 25813 & 27835 & 26631 & 29561 & 31527\end{array}$

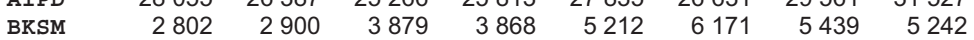

$\begin{array}{lllllllll}\text { BKSN } & 10743 & 8141 & 7077 & 3044 & 8498 & 11369 & 14834 & 37352\end{array}$

$\begin{array}{lllllllll}\text { BKSO } & 13698 & 14797 & 16797 & 18718 & 20993 & 23740 & 28327 & 21781\end{array}$

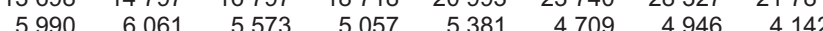

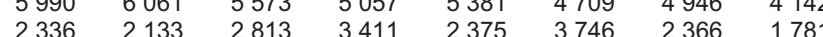

$\begin{array}{llllllll}2336 & 2133 & 2813 & 3411 & 2375 & 3746 & 2366 & 1781 \\ 1180 & 1586 & 2845 & 2457 & 2453 & 2378 & 2254 & 2166\end{array}$

BKSP

BKSQ

$64804 \quad 62005$

BKQJ

$\begin{array}{lllllllll}64804 & 62005 & 64250 & 62368 & 72747 & 78744 & 87727 & 103991\end{array}$

$\begin{array}{lllllllllll}\text { BKQK } & 314257 & 346034 & 381502 & 422065 & 461671 & 497806 & 621588 & 742317 & 889988\end{array}$

$\begin{array}{rr}43.0 & 52.9\end{array}$

4318

45822

18277

4244

2122

2284

Public sector net debt

RUTO

Source: Office for National Statistics: 02070142124

1 See chapter text.

2 Including overdraft with Bank of England.

3 Bank and building society deposits.

4 Gross domestic product at market prices from 12 months centred on the

end of the month. 
Current receipts

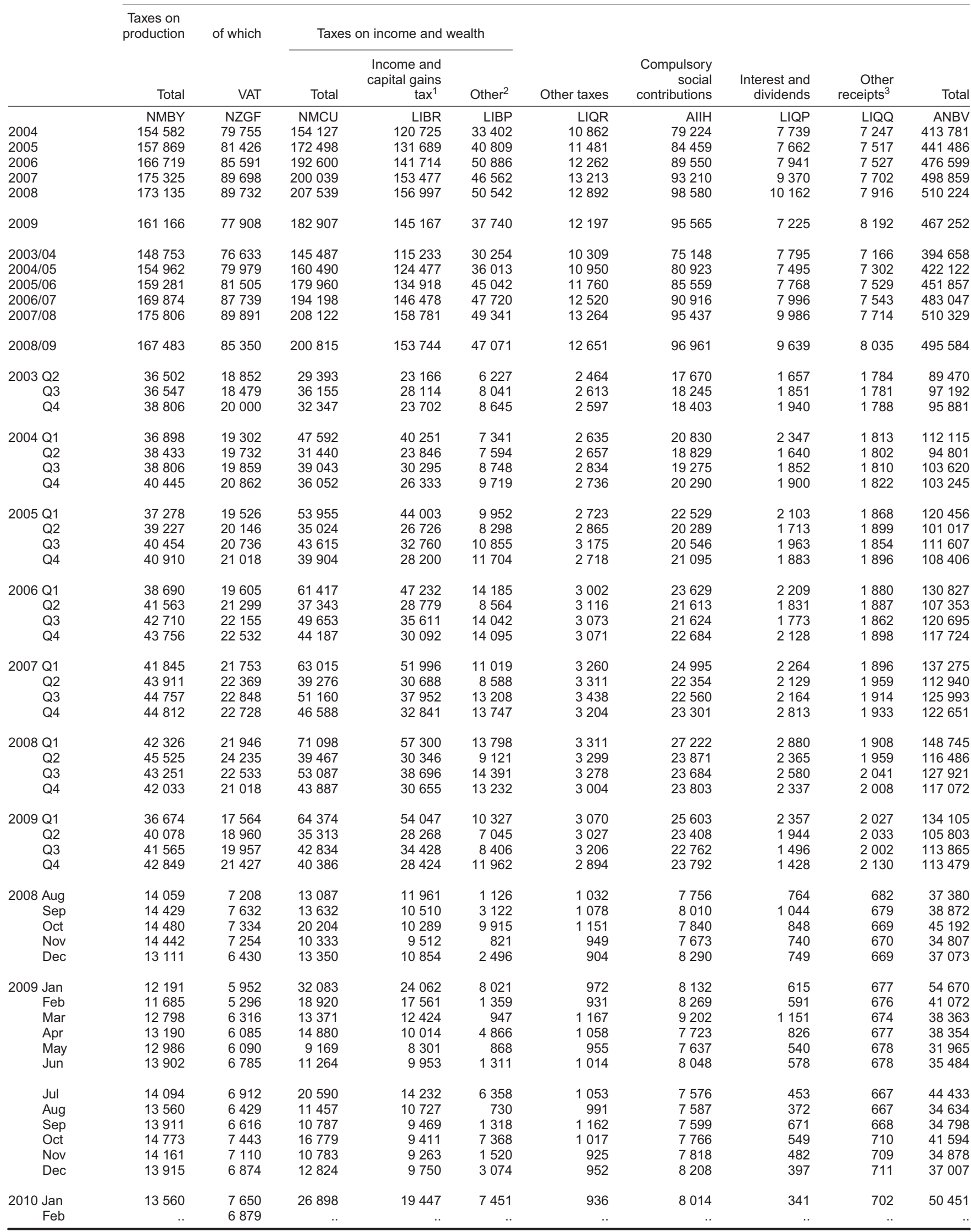




\begin{tabular}{|c|c|c|c|c|c|c|c|c|c|}
\hline & & Current $\epsilon$ & enditure & & & & & & \\
\hline & Interest & $\begin{array}{r}\text { Net Social } \\
\text { Benefits }\end{array}$ & Other & Total & $\begin{array}{r}\text { plus capital } \\
\text { taxes }\end{array}$ & Depreciation & $\begin{array}{l}\text { Surplus on } \\
\text { current budget }\end{array}$ & Net investment & Net borrowing \\
\hline & NMFX & GZSJ & LIQS & ANLP & ANPM & NSRN & ANLV & -ANNS & -NMFJ \\
\hline 2004 & 22955 & 122033 & 281640 & 426628 & -12847 & 5495 & -18342 & 18955 & 37297 \\
\hline 2005 & 25640 & 126132 & 301017 & 452789 & -11303 & 5711 & -17014 & 20409 & 37423 \\
\hline 2006 & 26666 & 130115 & 323791 & 480572 & -3973 & 5860 & -9833 & 25292 & 35125 \\
\hline 2007 & 30287 & 139097 & 332671 & 502055 & -3196 & 6071 & -9267 & 27801 & 37068 \\
\hline 2008 & 32247 & 148805 & 349958 & 531010 & -20786 & 6399 & -27205 & 39469 & 66674 \\
\hline 2009 & 25863 & 164482 & 368123 & 558468 & -91216 & 6637 & -100462 & 55765 & 156227 \\
\hline $2003 / 04$ & 22281 & 116926 & 267496 & 406703 & -12045 & 5514 & -17559 & 19570 & 37129 \\
\hline $2004 / 05$ & 23936 & 122624 & 287806 & 434366 & -12244 & 5513 & -17757 & 20798 & 38555 \\
\hline $2005 / 06$ & 25808 & 127304 & 306506 & 459618 & -7761 & 5734 & -13495 & 20000 & 33495 \\
\hline $2006 / 07$ & 27580 & 131346 & 324035 & 482961 & 86 & 5881 & -5795 & 26834 & 32629 \\
\hline $2007 / 08$ & 29957 & 140725 & 339346 & 510028 & 301 & 6125 & -5824 & 32882 & 38706 \\
\hline $2008 / 09$ & 30507 & 153588 & 352699 & 536794 & -41210 & 6530 & -47675 & 44106 & 91781 \\
\hline 2003 Q2 & 5681 & 27835 & 67149 & 100665 & -11195 & 1374 & -12569 & 4108 & 16677 \\
\hline Q3 & 5268 & 28921 & 64769 & 98958 & -1766 & 1363 & -3129 & 4491 & 7620 \\
\hline Q4 & 6028 & 31080 & 66676 & 103784 & -7903 & 1375 & -9278 & 4166 & 13444 \\
\hline 2004 Q1 & 5304 & 29090 & 68902 & 103296 & 8819 & 1402 & 7417 & 6805 & -612 \\
\hline Q2 & 5515 & 29988 & 70342 & 105845 & -11044 & 1360 & -12404 & 4121 & 16525 \\
\hline Q3 & 5644 & 30431 & 70667 & 106742 & -3122 & 1355 & -4477 & 4002 & 8479 \\
\hline Q4 & 6492 & 32524 & 71729 & 110745 & -7500 & 1378 & -8878 & 4027 & 12905 \\
\hline 2005 Q1 & 6285 & 29681 & 75068 & 111034 & 9422 & 1420 & 8002 & 8648 & 646 \\
\hline Q2 & 6259 & 30201 & 75127 & 111587 & -10570 & 1436 & -12006 & -163 & 11843 \\
\hline Q3 & 6206 & 31101 & 75274 & 112581 & -974 & 1407 & -2381 & 4925 & 7306 \\
\hline Q4 & 6890 & 35149 & 75548 & 117587 & -9181 & 1448 & -10629 & 6999 & 17628 \\
\hline 2006 Q1 & 6453 & 30853 & 80557 & 117863 & 12964 & 1443 & 11521 & 8239 & -3282 \\
\hline Q2 & 6334 & 31795 & 82844 & 120973 & -13620 & 1454 & -15074 & 6054 & 21128 \\
\hline Q3 & 6433 & 32899 & 80410 & 119742 & 953 & 1458 & -505 & 5208 & 5713 \\
\hline Q4 & 7446 & 34568 & 79980 & 121994 & -4270 & 1505 & -5775 & 5791 & 11566 \\
\hline 2007 Q1 & 7367 & 32084 & 80801 & 120252 & 17023 & 1464 & 15559 & 9781 & -5778 \\
\hline 2008 Q1 & 7037 & 33712 & 87476 & 128225 & 20520 & 1518 & 19002 & 14862 & -4140 \\
\hline Q2 & 8542 & 36908 & 89760 & 135210 & -18724 & 1607 & -20344 & 5820 & 26164 \\
\hline Q3 & 7989 & 37838 & 85494 & 131321 & -3400 & 1630 & -5044 & 9305 & 14349 \\
\hline Q4 & 8679 & 40347 & 87228 & 136254 & -19182 & 1644 & -20819 & 9482 & 30301 \\
\hline 2009 Q1 & 5297 & 38495 & 90217 & 134009 & 96 & 1649 & -1468 & 19499 & 20967 \\
\hline Q2 & 7025 & 40566 & 95526 & 143117 & -37314 & 1669 & -39182 & 12097 & 51279 \\
\hline Q3 & 4438 & 41509 & 91613 & 137560 & -23695 & 1618 & -25450 & 9218 & 34668 \\
\hline Q4 & 9103 & 43912 & 90767 & 143782 & -30303 & 1701 & -34362 & 14951 & 49313 \\
\hline 2008 Jul & 3283 & 12689 & 27507 & 43479 & 8190 & 543 & 7641 & 1899 & -5742 \\
\hline Aug & 3385 & 12617 & 28314 & 44316 & -6936 & 543 & -7488 & 1553 & 9041 \\
\hline Sep & 1321 & 12532 & 29673 & 43526 & -4654 & 544 & -5197 & 5853 & 11050 \\
\hline Oct & 3511 & 12558 & 28095 & 44164 & 1028 & 548 & 476 & 2008 & 1532 \\
\hline Nov & 3273 & 14742 & 29207 & 47222 & -12415 & 548 & -12982 & 1975 & 14957 \\
\hline Dec & 1895 & 13047 & 29926 & 44868 & -7795 & 548 & -8313 & 5499 & 13812 \\
\hline 2009 Jan & 2759 & 13574 & 28782 & 45115 & 9555 & 550 & 9037 & 6276 & -2761 \\
\hline Feb & 2394 & 12104 & 27989 & 42487 & -1415 & 550 & -1937 & 5203 & 7140 \\
\hline Mar & 144 & 12817 & 33446 & 46407 & -8044 & 549 & -8568 & 8020 & 16588 \\
\hline Apr & 3085 & 13388 & 33259 & 49732 & -11378 & 556 & -12391 & 1585 & 13976 \\
\hline May & 3324 & 13599 & 30837 & 47760 & -15795 & 556 & -16086 & 3449 & 19535 \\
\hline Jun & 616 & 13579 & 31430 & 45625 & -10141 & 557 & -10705 & 7063 & 17768 \\
\hline Jul & 2723 & 14310 & 30072 & 47105 & -2672 & 539 & -3342 & 2572 & 5914 \\
\hline Aug & 1526 & 13476 & 30023 & 45025 & -10391 & 539 & -11158 & 2565 & 13723 \\
\hline Sep & 189 & 13723 & 31518 & 45430 & -10632 & 540 & -10950 & 4081 & 15031 \\
\hline Oct & 3858 & 13796 & 30072 & 47726 & -6132 & 567 & -6627 & 2778 & 9405 \\
\hline Nov & 3506 & 15996 & 29769 & 49271 & -14393 & 567 & -15892 & 3044 & 18936 \\
\hline Dec & 1739 & 14120 & 30926 & 46785 & -9778 & 567 & -11843 & 9129 & 20972 \\
\hline 2010 Jan & 4267 & 13994 & 31231 & 49492 & 959 & 560 & .. & .. & .. \\
\hline
\end{tabular}

1 Includes capital gains tax paid by households. Includes income tax and 2 Mainly comprises corporation tax and petroleum revenue tax. 


\begin{tabular}{|c|c|c|c|c|c|c|c|c|c|c|}
\hline & & 2001 & 2002 & 2003 & 2004 & 2005 & 2006 & 2007 & 2008 & 2009 \\
\hline \multicolumn{11}{|l|}{ NATIONAL LOANS FUND² } \\
\hline Total assets & KQKD & 425955.6 & 434544.6 & 448006.3 & 108243.1 & 94226.5 & 83227.6 & 82872.1 & 89765.2 & 394715.0 \\
\hline $\begin{array}{l}\text { Total National Loans Fund } \\
\text { loans outstanding }\end{array}$ & $\mathrm{KQKE}$ & 51037.6 & 50251.4 & 47719.0 & 2963.1 & 2910.2 & 2964.2 & 3022.1 & 2970.5 & 2853.4 \\
\hline \multicolumn{11}{|l|}{ Loans to Public Corporations: } \\
\hline Royal Mail Group plc & KQKF & 500.0 & 500.0 & 550.0 & 500.0 & 500.0 & 500.0 & 500.0 & 500.0 & 500.0 \\
\hline Civil Aviation Authority & KQKQ & 92.5 & 9.8 & 8.8 & 8.2 & 7.6 & 11.0 & 10.1 & 9.1 & 8.0 \\
\hline British Railways Board & KQKS & 481.3 & & & & & & & & \\
\hline $\begin{array}{l}\text { British Waterways Board } \\
\text { New Towns - Development }\end{array}$ & KQ̃KU & 16.7 & 16.3 & 14.7 & 14.7 & 14.7 & 10.6 & 9.9 & 7.9 & 6.8 \\
\hline Royal Mint & KQLP & 5.0 & 14.8 & 11.3 & 15.7 & 18.1 & 22.5 & 14.9 & 8.1 & 7.4 \\
\hline Harbour Authorities & KQLV & 0.1 & 0.1 & 0.1 & 0.1 & 0.1 & 0.1 & 0.1 & 0.1 & 0.1 \\
\hline Ordnance Survey & GPVF & 13.9 & 12.3 & 11.0 & 9.9 & 8.9 & 8.0 & 7.3 & 6.7 & 6.2 \\
\hline Registers of Scotland & KZBB & 4.0 & 3.7 & 3.6 & 3.5 & 3.3 & 3.2 & 3.1 & 3.0 & 3.0 \\
\hline East of Scotland Water Authority & KZBC & 268.0 & 258.0 & 248.0 & 238.0 & 223.0 & 213.0 & 203.0 & 203.0 & 201.0 \\
\hline North of Scotland Water Authority & KZBD & 236.5 & 236.5 & 231.5 & 231.5 & 226.5 & 226.5 & 226.5 & 226.5 & 226.5 \\
\hline West of Scotland Water Authority & KZBE & 412.4 & 412.4 & 412.4 & 402.4 & 402.4 & 402.4 & 377.4 & 357.4 & 352.4 \\
\hline Loans to local authorities & KQLY & 47239.1 & 47093.4 & 44640.3 & 41468.3 & 42102.9 & 47123.7 & 48111.0 & 50753.0 & - \\
\hline \multicolumn{11}{|l|}{ Loans to private sector: } \\
\hline Housing associations & KGVS & 0.5 & .. & .. & .. &.. & .. &.. & .. & .. \\
\hline \multicolumn{11}{|l|}{$\begin{array}{l}\text { Loans within central government: } \\
\text { New Towns - Development }\end{array}$} \\
\hline Corporations and Commission & KQLD & 8.0 & 8.0 & 7.9 & 7.9 & 7.9 & 7.9 & 7.9 & 7.9 & 7.9 \\
\hline Scottish Homes & KQLF & 161.6 & 149.7 & 138.1 & 100.6 & - & .. & .. & .. & .. \\
\hline Housing Corporation (England) & $\mathrm{KQLH}$ & 3.0 & 2.0 & 2.0 & 1.2 & 1.4 & 1.4 & 1.4 & 1.4 & - \\
\hline Welsh Development Agency & KQLN & 0.3 & 0.2 & 0.1 & & .. & .. & .. & .. & .. \\
\hline Development Board for Rural Wales & KQLO & 4.0 & 4.0 & 4.0 & 4.0 & 4.0 & 4.0 & 3.9 & 3.9 & 3.9 \\
\hline $\begin{array}{l}\text { Northern Ireland Exchequer } \\
\text { Married quarters for Armed }\end{array}$ & KGVW & 1533.1 & 1473.9 & 1380.4 & 1372.0 & 1440.5 & 1503.5 & 1608.3 & 1589.1 & 1485.8 \\
\hline $\begin{array}{l}\text { Forces } \\
\text { Other assets. }\end{array}$ & KGVX & 57.7 & 56.4 & 54.9 & 53.4 & 51.8 & 50.1 & 48.3 & 46.4 & 44.4 \\
\hline \multicolumn{11}{|l|}{ Other assets: } \\
\hline $\begin{array}{l}\text { Account - Advances o/s } \\
\text { Subscriptions and contributions } \\
\text { to international financial } \\
\text { organisations: }\end{array}$ & KGVZ & 5680.0 & 831.0 & 30.0 & 670.0 & 910.0 & 2005.0 & 1805.0 & 1330.0 & 760.0 \\
\hline $\begin{array}{l}\text { International Monetary Fund } \\
\text { Borrowing included in public sector }\end{array}$ & KGXE & 9496.6 & 9494.5 & 9293.8 & 8696.8 & 8615.9 & 8813.5 & 8271.3 & 8880.8 & 11195.9 \\
\hline $\begin{array}{l}\text { net debt but not brought to } \\
\text { account by } 31 \text { March }\end{array}$ & KGXF & 405.9 & 417.5 & 467.1 & & & & & & \\
\hline Other NLF Assets & GLX9 &.. & .. & .. & 18545.9 & 18792.0 & 20735.2 & 20859.0 & 25180.0 & 36261.4 \\
\hline Debt Management Account & & & & & & & & & & \\
\hline -advances outstanding & GPVG & 35000.0 & 35000.0 & 28000.0 & 35000.0 & 20000.0 & - & & - & 292000.0 \\
\hline Consolidated Fund liability & KCYI & 324335.5 & 338550.2 & 362496.5 & 395161.4 & 436345.0 & 483836.2 & 519312.1 & 571228.4 & 730371.4 \\
\hline $\begin{array}{l}\text { Total liabilities } \\
\text { National Loans Fund - Gross }\end{array}$ & & & & & & & & & & \\
\hline liabilities outstanding & KCYJ & 425955.59 & 434544.59 & 448006.31 & 503404.50 & 530571.50 & 567063.81 & 602184.19 & 660993.56 & 11250856.00 \\
\hline $\begin{array}{l}1 \text { See Chapter text. } \\
2 \text { From 2003-04 the NLF Account has } \\
\text { The figures from } 2004 \text { onwards refle } \\
3 \text { Restated from } 2004 \text { onward. PWLB } \\
\text { loans. }\end{array}$ & $\begin{array}{l}\text { ect beer } \\
\text { ect this } \\
\text { advar }\end{array}$ & $\begin{array}{l}\text { epared on } \\
\text { counting che } \\
\text { s no longer }\end{array}$ & $\begin{array}{l}\text { an Accruals } \\
\text { lange. } \\
\text { included witl }\end{array}$ & $\begin{array}{l}\text { basis. } \\
\text { th NLF }\end{array}$ & & & & Source: $H$ & HM Treasury: & 02072704761 \\
\hline
\end{tabular}


Generation of income

Uses

Taxes on production and imports Taxes on products and imports Value added tax (VAT)

Paid to central government

Paid to the European Union

Total

Taxes and duties on imports excluding VAT

Paid to EU: import duties

Taxes on products excluding VAT and import duties Paid to central government

Customs and Excise revenue

Beer

Wines, cider, perry \& spirits

Tobacco

Hydrocarbon oils

Betting, gaming \& lottery

Air passenger duty

Insurance premium tax

Landfill tax

Other

Fossil fuel levy

Gas levy

Stamp duties

Camelot payments to National Lottery Distribution Fund

Hydro-benefit

Aggregates Levy

Climate change levy

Renewable energy obligations

Other taxes and levies

Total paid to central government

Paid to the European Union

Sugar levy

Total paid to the European Union

Total taxes on products excluding VAT \& import duties QYRA

Total taxes on products and imports

Production taxes other than on products

Paid to central government

Consumer Credit Act fees

National non-domestic rates

Old style non-domestic rates

Levies paid to CG levy-funded bodies

Motor vehicle duties paid by businesses

Regulator fees

Total

Paid to local government

Old style non-domestic rates

Total production taxes other than on products

Total taxes on production and imports, paid

Paid to central government

Paid to local government

Paid to the European Union
FJKM

QYRC

FUWE

NZGW

GTAN

GTAO

GTAP
CJOY

CJQY

CWAD

BKOF

ACDN

CIQY

GTAZ

GTBC

LIYH

LITN

MDUQ

LSNT

EP89

NMBV

$\overline{49768} \overline{51382} \overline{50551} \overline{52486} \overline{53664} \overline{56183} \overline{57965} \overline{61268} \overline{64434} \overline{58989}$

GTBA

FJWG

$\begin{array}{rlrllllllll}58688 & 60746 & 64735 & 69087 & 76633 & 79979 & 81505 & 87739 & 89891 & 85350\end{array}$

\begin{tabular}{llllllllll}
3451 & 4172 & 3592 & 2518 & 2574 & 1905 & 1964 & 2288 & 2571 & 2455 \\
\hline
\end{tabular}

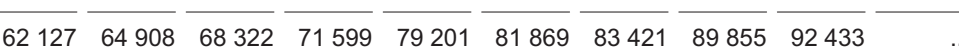

$\begin{array}{llllllllll}2049 & 2103 & 2024 & 1893 & 1957 & 2207 & 2264 & 2332 & 2462 & 2667\end{array}$

$\begin{array}{lllllllll}2848 & 2798 & 2907 & 2952 & 3084 & 3099 & 3092 & 3068 & 3034\end{array}$

$\begin{array}{lllllllll}3652 & 3814 & 4068 & 4430 & 4526 & 4790 & 4784 & 4846 & 5181\end{array}$

$\begin{array}{lllllllll}7796 & 7638 & 7639 & 8046 & 8092 & 8113 & 7952 & 8146 & 8006\end{array}$

$\begin{array}{lllllllll}22510 & 22630 & 21916 & 22147 & 22780 & 23313 & 23438 & 23585 & 24905\end{array}$

$\begin{array}{rrrrrrrrr}1500 & 1517 & 1317 & 977 & 898 & 876 & 884 & 958 & 961 \\ 882 & 956 & 802 & 804 & 799 & 872 & 906 & 1114 & 1949\end{array}$

$\begin{array}{rrrrrrrrr}882 & 956 & 802 & 804 & 799 & 872 & 906 & 1114 & 1949 \\ 1511 & 1751 & 1921 & 2189 & 2313 & 2353 & 2349 & 2317 & 2314\end{array}$

$456-475-501$

$\begin{array}{llrrrrrrr}- & - & - & - & - & 573 & 754 & 837 & 898\end{array}$

$\begin{array}{lllllllll}84 & 52 & - & - & - & - & - & - & - \\ - & - & 9 & - & - & - & - & -\end{array}$

$\begin{array}{llllllllll}6898 & 816 \overline{5} & 698 \overline{3} & 7549 & 7544 & 8966 & 10918 & 13386 & 14123\end{array}$

$\begin{array}{rrrrrrrrr}1593 & 1542 & 1520 & 1382 & 1311 & 1354 & 1397 & 1366 & 1349 \\ 38 & 44 & 44 & 44 & 43 & 40 & & \end{array}$

$\begin{array}{rrrrrrrrr}38 & 44 & 44 & 44 & 43 & 40 & - & - & - \\ - & - & - & 293 & 341 & 326 & 323 & 327 & 340\end{array}$

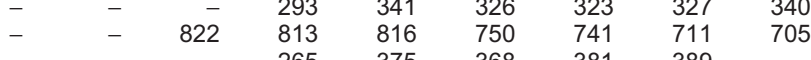

$\frac{46}{46} \frac{43}{43} \frac{27}{27} \frac{25}{25} \frac{19}{19} \frac{24}{24} \frac{24}{24} \frac{-}{-}-\frac{-}{-}-$

$49814 \overline{51425} \overline{50578} \overline{52511} \overline{53643} \overline{55989} \overline{57945} \overline{61050} \overline{64415}$

113990118436120924126003134801140065143630153236159313

$\begin{array}{lrrrrrrrrr}\text { CUDB } & 156 & 171 & 157 & 200 & 211 & 223 & 189 & 234 & 328\end{array}$

$\begin{array}{llllllllllllll}\text { CUKY } & 14353 & 15154 & 16252 & 16728 & 16902 & 17206 & 18147 & 19168 & 19584 & 21072\end{array}$

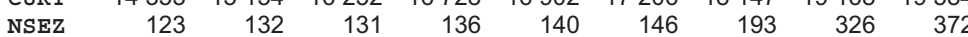

$\begin{array}{llllllllll}\text { LITK } & 234 & 213 & 215 & 190 & 194 & 218 & 239 & 244 & 256\end{array}$

$\begin{array}{llllllllll}\text { EKED } & 1559 & 1230 & 751 & 736 & 787 & 802 & 850 & 869 & 880\end{array}$

$\begin{array}{llllllllllll}\text { GCSQ } & 86 & 105 & 95 & 94 & 101 & 88 & 74 & 71 & 75 & . .\end{array}$

NMBX

$\begin{array}{llllllllll}16511 & 17005 & 17601 & 18084 & 18360 & 18802 & 19793 & 20912 & 21484 & 22990\end{array}$

$\begin{array}{lllllllllll}\text { NMYH } & 144 & 150 & 161 & 176 & 181 & 167 & 187 & 207 & 229 & 245\end{array}$

NMYD $\overline{16655} \overline{17155} \overline{17762} \overline{18260} \overline{18541} \overline{18969} \overline{19980} \overline{21119} \overline{21713} \overline{23440}$

NMBY 124727129536133199140152148753154962159281169874175806167483

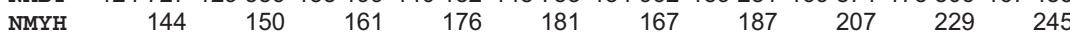

$\begin{array}{llllllllllll}\text { FJWB } & 5546 & 6318 & 5643 & 4436 & 4550 & 4136 & 4252 & 4620 & 5033 & 5122\end{array}$

NZGX $130645135591138686 \overline{144263} \overline{153367} \overline{159094} \overline{163486} \overline{174793} \overline{181032}$

$\begin{array}{rrrrrrrrr}- & - & - & 265 & 375 & 368 & 381 & 389 & . . \\ - & - & - & - & - & - & - & - & -\end{array}$




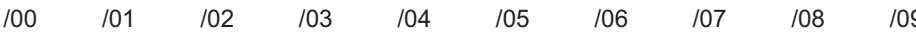

Secondary distribution of income

Uses

Current taxes on income, wealth etc

Taxes on income

Paid to central government

Household income taxes

Petroleum revenue tax

Windfall tax

Other corporate taxes

Total

DRWH

DBHA

EYNK

BMNX

NMCU

Other current taxes

Paid to central government

Motor vehicle duty paid by households

Old style domestic rates

Licences

Passport fees

Television licence fee

Total

Paid to local government

Old style domestic rates

Council tax

Total

Total

Total current taxes on income, wealth etc

Paid to central government

Paid to local government

Total

Social contributions

Actual social contributions

Paid to central governmen

(National Insurance Contributions)

Employers' compulsory contributions

Employees' compulsory contributions

Self- and non-employed persons' compulsory contributions

Total

Capital account

Changes in liabilities and net worth

Other capital taxes

Paid to central government

Inheritance tax

Tax on other capital transfers

Development land tax and other

Total

Total taxes and

compulsory social contributions

Paid to central government

Paid to local government

Paid to the European Union

Total

NMHK

NMHM

NMIS

NVCM

NMDE

AIIH
96977106866108526110407112356121273130555141226152194144443 $\begin{array}{llllllllll}853 & 1518 & 1310 & 958 & 1179 & 1284 & 2016 & 2155 & 1680 & 2567\end{array}$

$\begin{array}{rrrrrrrrr}184 \overline{2} & 345 \overline{8} & 330 \overline{2} & 265 \overline{7} & 3974 & 444 \overline{5} & 5539 & 700 \overline{6} & 8029\end{array}$

133994144263145185143256145487160490179960194198208122200815

\begin{tabular}{|c|c|c|c|c|c|c|c|c|c|}
\hline 3296 & 3039 & 3540 & 3600 & 3902 & 3935 & 4100 & 4270 & 4513 & 46 \\
\hline 117 & 108 & 109 & 104 & 129 & 227 & 235 & 247 & 272 & \\
\hline 8 & 2 & - & - & - & - & - & - & - & \\
\hline 1002 & 997 & 1065 & 1013 & 1008 & 1093 & 1221 & 1274 & 1313 & \\
\hline 89 & 113 & 139 & 153 & 198 & 237 & 285 & 346 & .. & \\
\hline 2286 & 2064 & 2183 & 2287 & 2391 & 2508 & 2623 & 2734 & .. & \\
\hline & & & & & & 484 & 02 & 174 & \\
\hline
\end{tabular}

$\begin{array}{lllllllll}68 & 76 & 80 & 85 & 92 & 111 & 149 & 157 & 173\end{array}$

$\begin{array}{rrrrrrrrr}68 & 76 & 80 & 85 & 92 & 111 & 149 & 157 & 173 \\ 12918 & 14155 & 15371 & 16809 & 18911 & 20190 & 21227 & 22299 & 23398\end{array}$

$\begin{array}{llllllllll}12986 & 14231 & 15451 & 16894 & 19016 & 20335 & 21375 & 22497 & 23677 & 24729\end{array}$

$\overline{19784} \overline{20554} \overline{22487} \overline{24051} \overline{26649} \overline{28354} \overline{29859} \overline{31399} \overline{33051} \overline{34446}$

NMCP $\quad 140792150586152215150447153191168509188444203400217662210532$

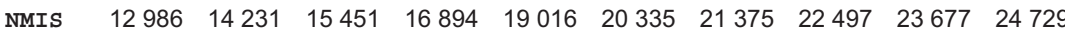

NMZL $\quad \overline{153778} \overline{164817} \overline{167666} \overline{167341} \overline{172207} \overline{188844} 209819225897241339235261$

$\begin{array}{lllllllllllll}\text { CEAN } & 31705 & 35212 & 35816 & 35476 & 41459 & 44864 & 47425 & 50356 & 54030 & 55952\end{array}$ $\begin{array}{llllllllllll}\text { GCSE } & 23289 & 24772 & 25130 & 25701 & 31013 & 33088 & 35181 & 37426 & 39250\end{array}$

$\begin{array}{llllllllll}1941 & 2084 & 2216 & 2352 & 2676 & 2744 & 2852 & 2956 & 3032 & 3112\end{array}$ $\overline{56935} \overline{62068} \overline{63162} \overline{63529} \overline{75148} \overline{80923} \overline{85559} \overline{90916} \overline{95437} \overline{96961}$

\begin{tabular}{|c|c|c|c|c|c|c|c|c|c|}
\hline GILF & 2016 & 2181 & 2346 & 2323 & 2486 & 2874 & 3226 & 3508 & 3814 \\
\hline GILG & 38 & 55 & 37 & 47 & 35 & 48 & 50 & 50 & 50 \\
\hline GCSV & - & - & - & - & - & - & - & - & - \\
\hline MGI & 2054 & 2236 & 2383 & 2370 & 2521 & 2941 & 3276 & 3618 & 3890 \\
\hline
\end{tabular}

GCSS 324736344015350642355995379465406928436107467323492501

$\begin{array}{llllllllllll}\text { GCST } & 13130 & 14381 & 15612 & 17070 & 19195 & 20498 & 21563 & 22663 & 23800\end{array}$

$\begin{array}{lllllllllll}\text { FJWB } & 5546 & 6318 & 5643 & 4436 & 4550 & 4136 & 4252 & 4620 & 5033 & 5122\end{array}$

GCSU $\overline{343412} \overline{364714} \overline{371897} \overline{377501} \overline{403258} \overline{431975} \overline{462356} \overline{495079} \overline{520614} \overline{504909}$

Total taxes and social contributions as percentage of GDP

GDWM

$36.3 \quad 36.8$

36.0

34.5

34.8

35

$\begin{array}{lll}36.4 & 36.8 \quad 36.6\end{array}$

1 See chapter text.

Office for National Stat 


$\begin{array}{llllllllll}1999 & 2000 & 2001 & 2002 & 2003 & 2004 & 2005 & 2006 & 2007 & 2008\end{array}$

\section{Borrowing}

Government securities: new issues

National savings securities:

National savings certificates

Capital bonds

Income bonds

Deposit bonds

British savings bonds

Premium savings bonds

Residual Account

Save As You Earn

Yearly plan

National savings stamps and gift$$
\text { tokens }
$$

National Savings Bank Investments

Children's Bonus Bonds

First Option Bonds

Pensioners Guaranteed Income Bond

Treasurer's account

Individual Savings Accoun

Fixed Rate Savings Bonds

Guaranteed Equity Bonds

Easy Access Savings Account

Certificate of tax deposit

Nationalised industries', etc

temporary deposits

Sterling Treasury bills (net receipt)

ECU Treasury bills (net receipt)

ECU Treasury notes (net receipt)

Ways and means (net receipt)

Other debt : payable in sterling :

Interest free notes

Other debt: payable in external currencies

Total receipts

\section{Repayment of debt}

Government securities: redemptions

Statutory sinking funds

Terminable annuities:

National Debt Commissioners

National savings securities:

National savings certificates

Capital bonds

Income bonds

Deposit bonds

Yearly Plan

British savings bonds

Premium savings bonds

Residual Account

Save As You Earn

National savings stamps and gift tokens

National Savings Bank Investments (repayments)

Children's Bonus Bonds

First Option Bonds

Pensioners Guaranteed Income Bond

Treasurer's account

Individual Savings Account

Fixed Rate Savings Bonds

Guaranteed Equity Bonds

Easy Access Savings Account

Certificates of tax deposit

Tax reserve certificates

Nationalised industries', etc

temporary deposits

Debt to the Bank of England

Sterling Treasury bills (net repayment)

ECU Treasury bills (net repayment)

ECU Treasury notes (net repayment)

Ways and means (net repayment)

Other debt: payable in sterling :

Interest free notes

Other

KQGA

KQGB

KQGC

KQGD

KQGF

KQGG

JT3F

KQGH

KQGI

KOGJ

KQGK

KGVO

KIAR

KJDW

KWNF

ZAFC

ECPU

C30M

KQGL

KQGM

KQGO

KOGP

KDZZ

KQGQ

KOGR

$\mathrm{HCZ}$

KQGS

KQGT

KQGU

KQGV

KQGW

KQGX

KQGY

KQGZ

KQHA

JT3G

$\mathrm{KQHC}$

KQHD

KQHE

KGVQ

KIAS

KPOB

KWNG

ZAFE

ZAFF

JUWE

$\mathrm{KQHF}$

$\mathrm{KQHH}$

$\mathrm{KPOC}$

KQHJ

KJEG

KSPA

$\mathrm{KQHK}$

KOHL

$\mathrm{KQHM}$

Other debt : payable in external currencies KQHN

Total payments

КQHO

Net borrowing

Net repayment KHDD
$26426.525789 .843433 .4 \quad 54068.9 \quad 53220.9 \quad 57290.5 \quad 80668.9 \quad 66233.4 \quad 64197.4 \quad 276504.4$

$\begin{array}{rrrrrr}1962.7 & 3086.2 & 2580.7 & 2434.3 & 1940.4 & 1696.4 \\ 35.40 & 29.00 & 40.90 & 107.30 & 65.00 & 25.20 \\ 653.4 & 760.5 & 625.6 & 484.8 & 415.3 & 426.6 \\ . . & . . & . . & . . & - & . . \\ . . & . . & . . & . . & - & . \\ 3449.4 & 3296.0 & 3859.6 & 4604.5 & 7530.1 & 5737.8 \\ & & & & & \\ . . & . . & . . & . . & . . & . . \\ 5.0 & 0.3 & . . & . . & - & . . \\ . . & . . & . . & . . & - & . . \\ . . & & & & & \\ 901.6 & 955.3 & 864.9 & 1012.4 & 809.9 & 817.5 \\ 58.5 & 53.4 & 45.0 & 54.0 & 51.7 & 66.8 \\ 34.3 & . . & . . & . . & - & . . \\ 590.7 & 687.2 & 603.5 & 662.9 & 274.2 & 323.9 \\ 13.6 & 12.5 & 15.2 & 19.4 & 13.9 & 11.1 \\ 257.8 & 265.9 & 397.8 & 405.6 & 335.4 & 276.4 \\ 175.9 & 284.7 & 192.7 & 193.0 & 82.0 & 86.3 \\ . . & . . & 27.2 & 274.8 & 227.9 & 317.1 \\ . . & . . & . . & . . & 126.9 & 903.5 \\ 121.4 & 76.5 & 77.6 & 59.6 & 145.2 & 114.8\end{array}$

1206.8

$34.30 \quad 20.70 \quad 2524.9$

$\begin{array}{lll}567.5 & 593.5 & 1502.6\end{array}$

7817.0

8432.

6573.2

8472.2

343.3

110.6

643.6
59.5

558.

142.7

216.4

11.6

10.9
261.3

1015.1

69.5

513.2

100.2

535.7

371.3

1071.8

46.8

0.3

1394.2

347.4

56.0

163.7

835.0

4327.6

99.9

3763.5

1301.7
721.1

$\begin{array}{lllllll}5599.0 & 12126.0 & 12095 . \ddot{3} & 3899 . \ddot{9} & 22700 . \overline{2}\end{array}$

$23428.0 \quad 12810.0$

\begin{tabular}{|c|c|c|c|c|c|c|c|c|c|}
\hline 373.5 & 972.7 & 1427.2 & 754.0 & 1213.2 & 662.3 & 1858.9 & 1049.9 & 97.2 & 822.8 \\
\hline .. & .. & .. & .. & 1792.5 & .. & .. & .. & .. & . \\
\hline 31723 & 104503 & 128437 & 124430 & 138903 & 93778 & 116163 & 139047 & 142961 & 364394 \\
\hline
\end{tabular}

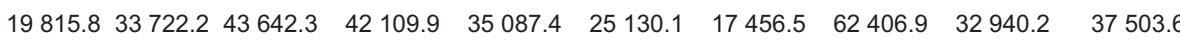

2.0

64.3
1.9

1.9

1.8

1.8

0.4

$2405.2 \quad 4546.8 \quad 4177.7$

$324.2 \quad 375.0 \quad 175.9$

$\begin{array}{lll}1686.3 & 857.0 \quad 933.8\end{array}$

4146.7

2769.1

1107.4

$1172.1 \quad 1201.9$

1240.2

$70.2-71.1-45.4$

4.4

121.1

159.2

172.1
137.4
719.2

184.0

229.0

2145.1

$141.8 \quad 18.4 \quad 4.5$

$1923.8 \quad 1872.6 \quad 1942.9$

0.8

4.9

19.3

4613.9

$\begin{array}{lrrrr}34.5 & 22 . \ddot{ } & 8.0 & 3 . \ddot{ } & 0.0\end{array}$

$0 . \ddot{5}$

14.9

1.2

$1886.3 \quad 1654.1 \quad 1415.8$

$1350.1 \quad 1342.7 \quad 1554.0$

$\begin{array}{rrr}69.3 & 95.0 & 114.5 \\ 298.1 & 225.2 & 111.6\end{array}$

$935.302003 .80 \quad 1640.40$

$\begin{array}{lrr}16.4 & 13.9 & 16.5\end{array}$

$\begin{array}{lll}12.3 & 39.9 & 70.3\end{array}$

$\begin{array}{lll}2.8 & 62.1 & 110.1\end{array}$

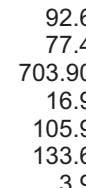

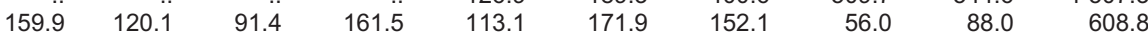

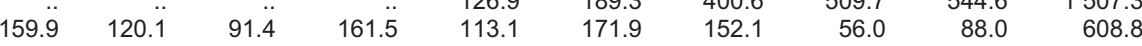

79.8
62.2

84.5

1172.4

976.8

1058.2

$\begin{array}{rrr}7 & 33.4 \\ 16.9 & 538.50 & 445.00\end{array}$

$\begin{array}{rrrrrr}14.2 & 16.2 & 18.3 & 11.7 & 47.1 & 18.6\end{array}$

95.8
36.1

105.7
25.6

108.5

106.1

$\begin{array}{rrrr}36.1 & 25.6 & 26.6 & 25.2\end{array}$

$\begin{array}{rrrrrr}157.6 & 202.2 & 194.1 & 193.6 & 274.9 & 822.2\end{array}$

$\begin{array}{rrrrrr}153.1 & 92.1 & 105.0 & 77.2 & 104.2 & 246.8\end{array}$

$\begin{array}{llllll}3.3 & . . & 0.2 & 3.7 & 365.9 & 370.3\end{array}$

$\begin{array}{lllrrr}126.9 & 189.3 & 400.6 & 509.7 & 544.6 & 1507.3\end{array}$

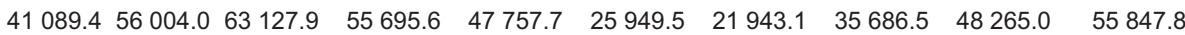

$3014 . \ddot{6} 6194.2$

2492.9

.. $1391 . \ddot{9} \quad 1359 . \ddot{6} \quad 1453 . \ddot{1}$

$9760.2 \quad 36207.3$

3161.7

$\begin{array}{llllllllll}246.4 & 458.2 & 1723.3 & 1393.3 & 990.5 & 300.4 & 222.3 & 586.4 & 474.4 & 1092.9\end{array}$

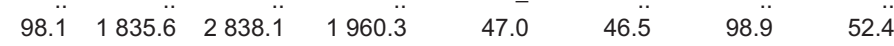

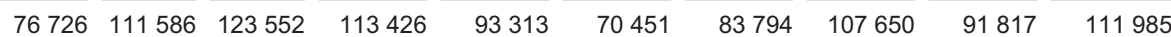

$\overline{4997.30} \overline{. . .} \overline{4884.70} \overline{11004.40} \overline{45590.10} \overline{23327.30} \overline{32369.30} \overline{31397.00} \overline{51143.80} \overline{252409.34}$

Note: the table excludes transactions in treasury bills issued for the Special 


\begin{tabular}{|c|c|c|c|c|c|c|c|c|c|c|c|c|c|}
\hline & \multicolumn{8}{|c|}{ Cash receipts } & \multicolumn{4}{|c|}{ Cash outlays } & \multirow{3}{*}{$\begin{array}{r}\text { Own } \\
\text { account } \\
\text { net cash } \\
\text { requireme- }\end{array}$} \\
\hline & \multicolumn{5}{|c|}{ HM Revenue and Customs } & \multirow[b]{2}{*}{$\begin{array}{r}\text { Interest } \\
\text { and } \\
\text { dividends }\end{array}$} & \multirow[b]{2}{*}{$\begin{array}{l}\text { Other } \\
\text { receipts }\end{array}$} & \multirow[b]{2}{*}{ Total } & \multirow{2}{*}{\multicolumn{2}{|c|}{$\begin{array}{r}\text { Net } \\
\text { acquisiti- } \\
\text { on of } \\
\text { company } \\
\text { securities } \\
6\end{array}$}} & \multirow[b]{2}{*}{$\begin{array}{r}\text { Net } \\
\text { depart- } \\
\text { mental } \\
\text { outlays }^{7}\end{array}$} & \multirow[b]{2}{*}{ Total } & \\
\hline & $\begin{array}{l}\text { Total } \\
\text { paid } \\
\text { over }^{1}\end{array}$ & $\underset{2}{\text { Income tax }}$ & $\begin{array}{l}\text { Corpora- } \\
\text { tion tax }{ }^{2}\end{array}$ & $\mathrm{NICs}{ }^{3}$ & V.A.T. ${ }^{4}$ & & & & & & & & \\
\hline & 1 & 2 & 3 & 4 & 5 & 6 & 7 & 8 & 9 & 10 & 11 & 12 & \\
\hline & MIZX & RURC & ACCD & ABLP & EYOO & RUUL & RUUM & RUUN & RUUO & ABIF & RUUP & RUUQ & RUU \\
\hline 2000 & 305547 & 103118 & 33003 & 59274 & 58509 & 9009 & 46078 & 360634 & 23890 & -251 & 297933 & 321572 & -3906 \\
\hline 2001 & 316517 & 111874 & 33520 & 62973 & 60282 & 8611 & 24643 & 349771 & 23132 & -661 & 324633 & 347104 & -2667 \\
\hline 2002 & 315987 & 111559 & 28866 & 63992 & 63000 & 6954 & 25310 & 348251 & 19343 & - & 347612 & 366955 & 1870 \\
\hline 2003 & 325138 & 113712 & 28489 & 69360 & 67525 & 7335 & 25329 & 357802 & 20348 & -39 & 379418 & 399727 & 4192 \\
\hline 2004 & 347514 & 121493 & 31160 & 77026 & 71907 & 6855 & 25137 & 379506 & 21027 & - & 400631 & 421658 & 4215 \\
\hline 2005 & 372567 & 130818 & 37820 & 83612 & 73012 & 6549 & 26341 & 405457 & 22434 & - & 421021 & 443455 & 37998 \\
\hline 2006 & 401362 & 140616 & 47108 & 87156 & 76103 & 6640 & 28115 & 436117 & 25834 & -347 & 448131 & 473618 & 37501 \\
\hline 2008 & 428380 & 157500 & 46487 & 98504 & 80709 & 9354 & 30556 & 468290 & 26033 & 19714 & 544720 & 590467 & 122177 \\
\hline 2009 & 384872 & 146721 & 35331 & 95411 & 68635 & 6666 & 31262 & 422800 & 29264 & 41809 & 548771 & 619844 & 19704 \\
\hline $1999 / 00$ & 291280 & 96032 & 34322 & 56354 & 56395 & 8637 & 22660 & 322577 & 24320 & -535 & 288128 & 311913 & -1066 \\
\hline $2000 / 01$ & 309726 & 108414 & 32421 & 60614 & 58501 & 8715 & 46772 & 365213 & 23798 & -81 & 304245 & 327962 & -3725 \\
\hline $2001 / 02$ & 314959 & 111028 & 32041 & 63168 & 61026 & 7843 & 25001 & 347803 & 22126 & -683 & 329726 & 351169 & 336 \\
\hline $2002 / 03$ & 317174 & 111102 & 29268 & 64553 & 63451 & 7425 & 24725 & 349324 & 19687 & -39 & 353890 & 373538 & 2421 \\
\hline $2003 / 04$ & 331133 & 116194 & 28077 & 72457 & 69075 & 7172 & 25348 & 363653 & 21251 & - & 385119 & 406370 & 4271 \\
\hline $2004 / 05$ & 355917 & 125202 & 33641 & 78098 & 73026 & 6633 & 25074 & 387624 & 21810 & - & 403268 & 425078 & 37454 \\
\hline $2005 / 06$ & 382067 & 133519 & 41829 & 85522 & 72856 & 6393 & 27022 & 415482 & 23121 & -347 & 428616 & 451390 & 35908 \\
\hline $2006 / 07$ & 406337 & 147134 & 44308 & 87274 & 77360 & 6754 & 27359 & 440450 & 26279 & - & 451062 & 477341 & 36891 \\
\hline $2007 / 08$ & 431800 & 152591 & 46383 & 100411 & 80601 & 9000 & 31205 & 472005 & 25390 & -2340 & 478576 & 501626 & 2962 \\
\hline $2008 / 09$ & 416512 & 155704 & 43077 & 96884 & 78439 & 8724 & 28008 & 453244 & 25947 & 32250 & 557560 & 615757 & 16251 \\
\hline 2007 Q3 & 107134 & 37488 & 12465 & 24165 & 19301 & 1986 & 9934 & 119054 & 6486 & - & 114418 & 120904 & 185 \\
\hline Q4 & 101691 & 31137 & 12957 & 22764 & 21327 & 2504 & 7070 & 111265 & 6473 & - & 124364 & 130837 & 1957 \\
\hline 2008 Q1 & 126971 & 54549 & 12946 & 27550 & 19850 & 2646 & 5997 & 135614 & 6472 & - & 118768 & 125240 & -1037 \\
\hline Q2 & 97153 & 34333 & 8509 & 23517 & 20087 & 2252 & 8154 & 107559 & 6449 & - & 131441 & 137890 & 3033 \\
\hline 2009 Q1 & 115103 & 52753 & 9536 & 25930 & 17580 & 2016 & 3449 & 120568 & 6386 & 12536 & 131608 & 150530 & 2996 \\
\hline Q2 & 85700 & 31145 & 6338 & 22729 & 16104 & 1892 & 9631 & 97223 & 8516 & -2021 & 145067 & 151562 & \\
\hline Q3 & 93410 & 35190 & 8026 & 23863 & 16846 & 1357 & 9713 & 104480 & 7584 & - & 133138 & 140722 & 3624 \\
\hline Q4 & 90659 & 27633 & 11431 & 22889 & 18105 & 1401 & 8469 & 100529 & 6778 & 31294 & 138958 & 177030 & 7650 \\
\hline
\end{tabular}

Relationships between columns $1+6+7=8 ; 9+10+11=12 ; 12-8=13$

5 Including some elements of expenditure not separately identified.

1 Comprises payments into the Consolidated Fund and all payovers of NICS 6 Mainly comprises privatisation proceeds.

excluding those for Northern Ireland.

2 Income tax includes capital gains tax and is neto
HM Revenue and Customs as tax deductions.

3 UK receipts net of personal pension rebates; gross of Statutory Maternity

7 Net of certain receipts, and excluding on-lending to local authorities and public corporations.

Pay and Statutory Sick Pay.

Sources: HM Revenue \& Customs Office for National Statistics

4 Payments into Consolidated Fund. 


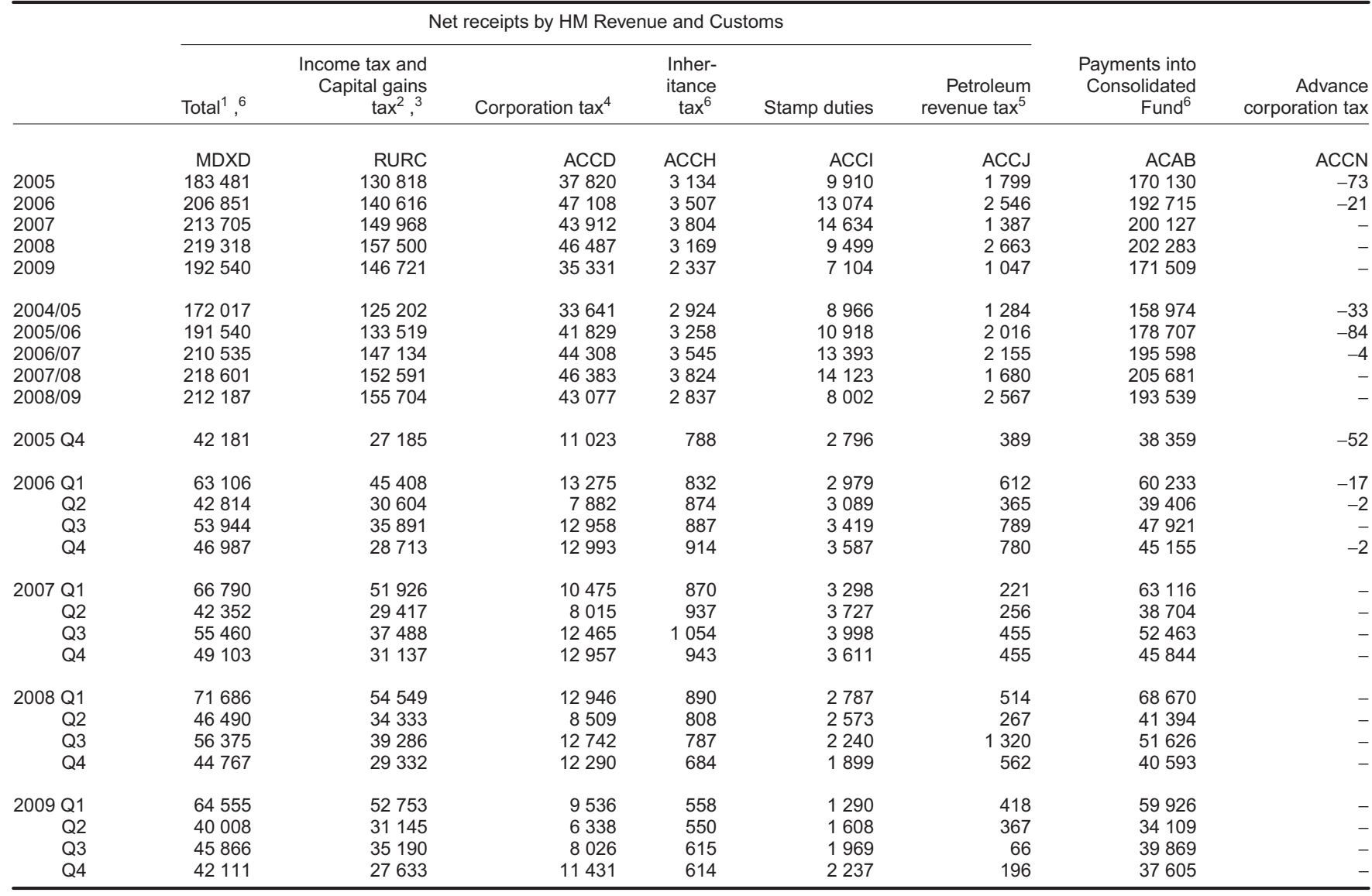

1 The total is not always equal to the sum of the individual taxes due to 5 Including net advance petroleum revenue tax.

rounding.

2 Income tax and Capital gains tax combined.
3 Figures for income tax treat payments of the personal tax credits as negative tax to the extent that the credits are less than or equal to the tax liability of the family. Payments exceeding this liability are treated as public expenditure.

4 Including net advance corporation tax receipts shown separately in the final column.

6 Payments into the consolidated fund are not directly comparable to receipts Over the year payments into the consolidated fund will always be lower than total receipts because the public expenditure element of payments of tax being recorded in receipts. Because the public expenditure element of payments of tax credits (both personal and company) are deducted from the payments into the consolidated fund but have no impact on receipts. In addition, there is a timing difference between payments taking value and hence paid over to the consolidated fund and being recorded in receipts.

Sources: HM Revenue and Customs; National Statistics

\section{British government and government guaranteed marketable securities ${ }^{1}$ Nominal values of official and market holdings by maturity ${ }^{2,3}$ \\ At 31 March each year}

$£$ million

\begin{tabular}{|c|c|c|c|c|c|c|c|c|c|c|c|c|}
\hline & & 1999 & 2000 & 2001 & 2002 & 2003 & 2004 & 2005 & 2006 & 2007 & 2008 & 2009 \\
\hline Total holdings & KQMO & 291788 & 290629 & 285915 & 278808 & 292777 & 321051 & 354884 & 411770 & 442857 & 478779 & 71322 \\
\hline Up to 5 years & KQMP & 95112 & 95131 & 92090 & 92780 & 106074 & 88678 & 110839 & 122496 & 119872 & 117620 & 21188 \\
\hline Over 5 and up to 15 years & KQMQ & 124603 & 116910 & 120101 & 106044 & 101465 & 131665 & 123729 & 151841 & 167525 & 168623 & 21068 \\
\hline Over 15 years (including undated) & KQMR & 72074 & 78587 & 73724 & 79984 & 85238 & 97500 & 117350 & 134485 & 152529 & 192536 & 29065 \\
\hline \multicolumn{13}{|l|}{ Official holdings: ${ }^{3}$} \\
\hline Total & HHAW & 6394 & 6204 & 8210 & 7558 & 10650 & 9118 & 7433 & 25409 & 23305 & 25754 & 133080 \\
\hline Up to 5 years & HHAY & 2600 & 2849 & 4652 & 3928 & 4797 & 3321 & 2770 & 8222 & 7328 & 7120 & 4099 \\
\hline Over 5 and up to 15 years & HHAZ & 2989 & 2567 & 3009 & 2844 & 4115 & 4015 & 3063 & 9620 & 9511 & 10329 & 3980 \\
\hline Over 15 years (including undated) & HHBA & 805 & 788 & 549 & 786 & 1738 & 1540 & 1562 & 7530 & 6420 & 8304 & 5228 \\
\hline \multicolumn{13}{|l|}{ Market holdings: } \\
\hline Total & HHBB & 285394 & 284425 & 277705 & 271250 & 282127 & 311933 & 347451 & 386361 & 419552 & 453025 & 58014 \\
\hline Up to 5 years & HHBD & 92512 & 92282 & 87438 & 88852 & 101277 & 85357 & 108070 & 114274 & 112545 & 110500 & 17089 \\
\hline Over 5 and up to 15 years & HHBE & 121614 & 114343 & 117092 & 103200 & 97350 & 127650 & 120666 & 142221 & 158014 & 158294 & 17087 \\
\hline Over 15 years (including undated) & HHBF & 71269 & 77800 & 73175 & 79198 & 83500 & 95960 & 115788 & 126955 & 146109 & 184231 & 23836 \\
\hline
\end{tabular}

Over 15 years (including undated)

1 The government guaranteed securities of nationalised industries only. A reled.

2 Securities with optional redemption dates are classified according to the final redemption date. The nominal value of index-linked British Government Stock has been raised by the amount of accrued capital uplift.
3 Official holdings were changed following the introduction of the central bank sector in the UK national accounts. These holdings now principally include those of the Debt Management Office and other government departments. The Issue and Banking Departments of the Bank of England are classified within the central bank sector and are therefore part of market holdings. 


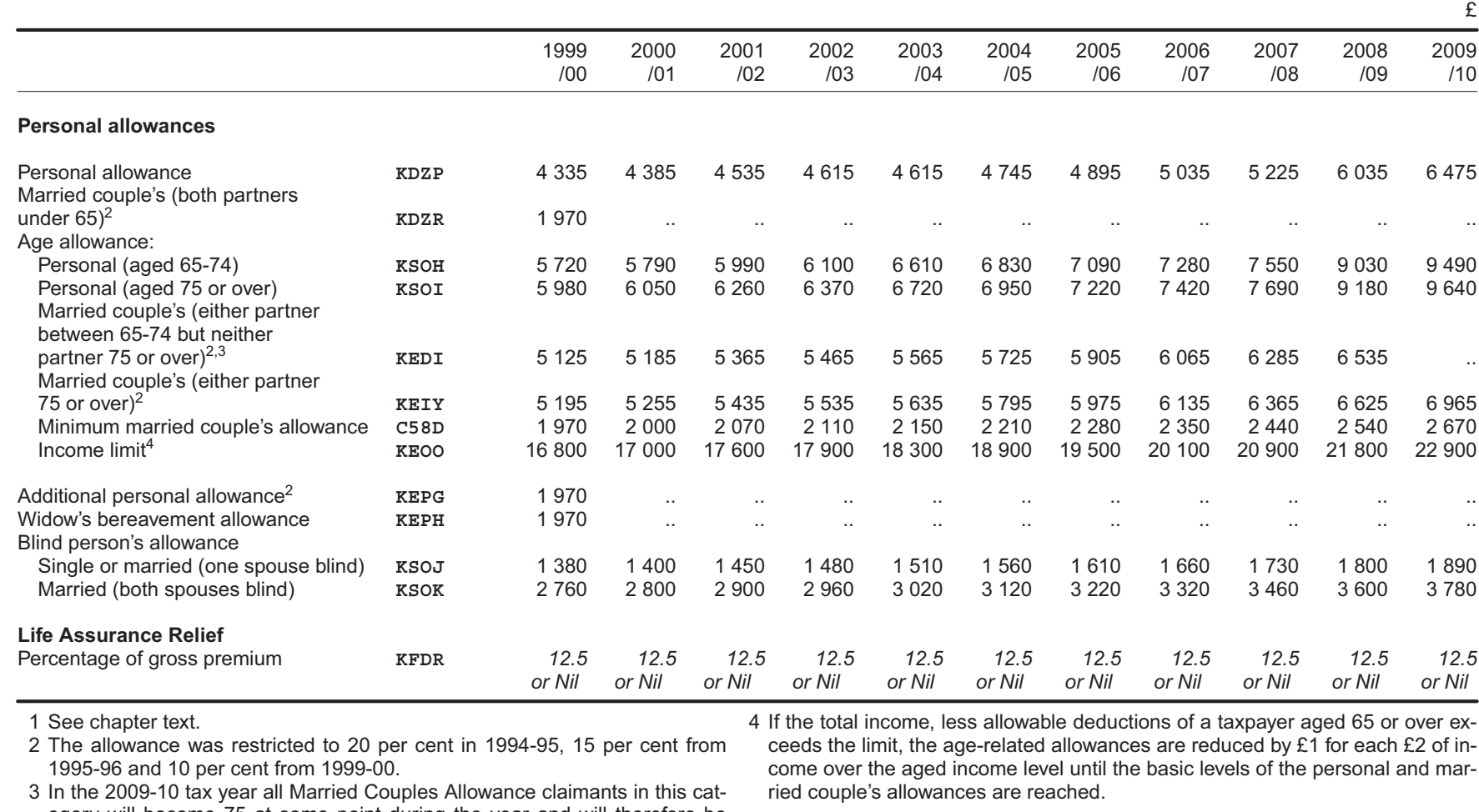

egory will become 75 at some point during the year and will therefore be entitled to the higher amount of allowance, for those aged 75 and over.

\subsection{Rates of income tax}

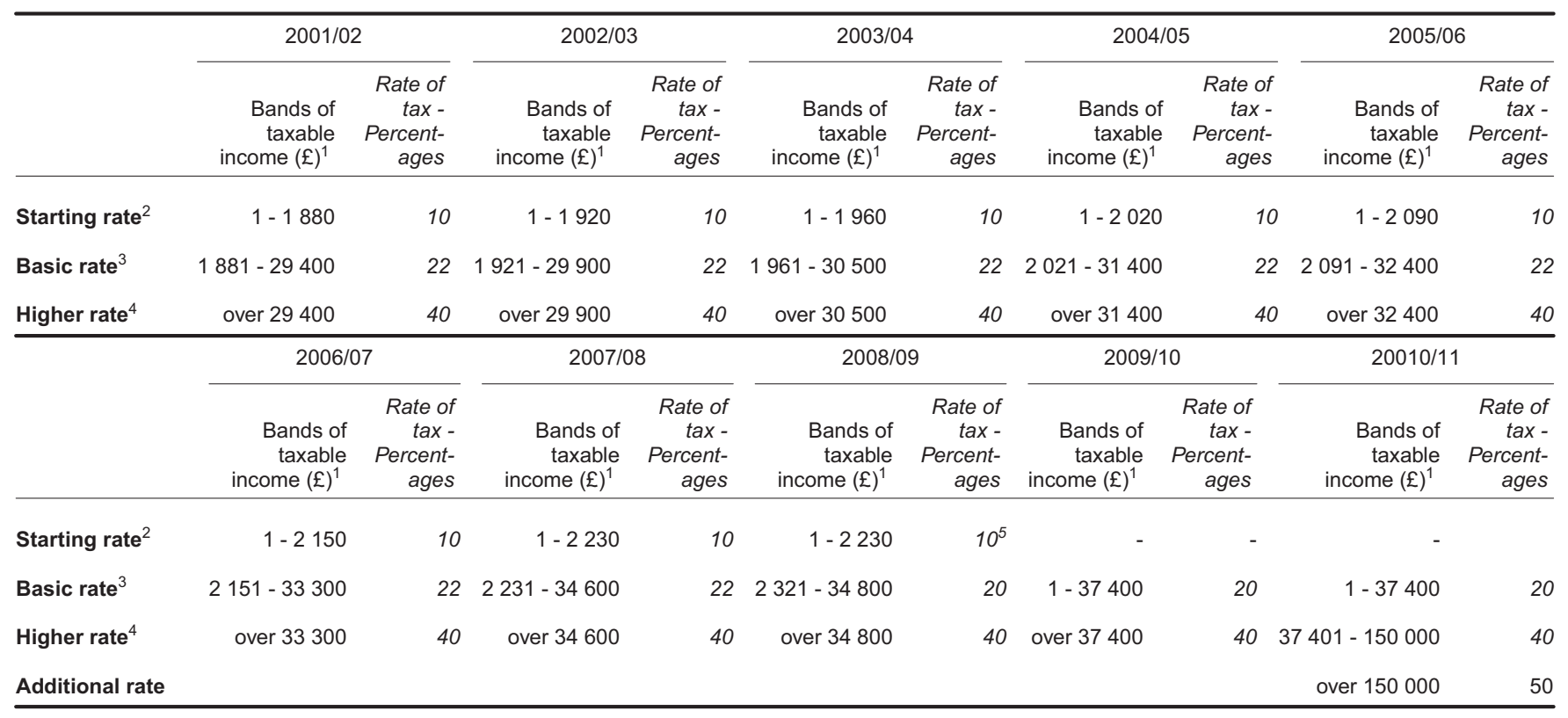

\footnotetext{
1 Taxable income is defined as gross income for income tax purposes less any allowances and reliefs available at the taxpayer's marginal rate.

2 The starting rate also applies to savings and dividends.

3 The basic rate of tax on dividends is $10 \%$ and savings income is $20 \%$.

4 The higher rate of tax on dividends is $32.5 \%$.

5 From $2008 / 09$ there is a $10 \%$ starting rate for savings income only. If nonsavings income is above this limit the $10 \%$ rate does not apply.
}

Source: HM Revenue \& Customs: 02071473045 
United Kingdom

\begin{tabular}{ll} 
Total debt & KQB \\
Public Works Loan Board & \\
Northern Ireland Consolidated Fund & KQB \\
Other debt & KQB \\
\hline
\end{tabular}

$\begin{array}{rr}52.9 & 59.7 \\ 42.4 & 47 \\ 0.3 & \\ 10.5 & \end{array}$

59.7

62.3

66.2

67.2

Other debt

KQBT

47.1

47.9

50.3

England

Total debt

of which Public Works Loan Board

$\mathrm{C} 300$

40.1

46.1

48.6

52.4

53.6

Wales

Total debt

of which Public Works Loan Board

C3OQ

3.7

3.8

3.8
3.1

40.2

Scotland

Total debt

KQBX

3.1

3.3

of which Public Works Loan Board

KQBY

8.7

9.4
7.2

9.5

9.4

9.4

Northern Ireland

Total debt

KQBZ

0.3

0.3

0.3

0.4

0.4

of which
Northern Ireland Consolidated Fund

KQBT

0.3

1 The sums shown exclude inter-authority loans.

Sources: Communities and Local Government: 0207944 4176; Public Works Loan Board: 0207862 6610,

\subsection{4 \\ Rateable Values ${ }^{1}$ \\ England and Wales \\ At 1 April each year}

\begin{tabular}{|c|c|c|c|c|c|c|c|c|c|c|c|c|}
\hline & & 1999 & 2000 & 2001 & 2002 & 2003 & 2004 & 2005 & 2006 & 2007 & 2008 & 2009 \\
\hline \multicolumn{13}{|c|}{ Number of properties (Thousands) } \\
\hline Commercial & KMIN & 1219 & 1223 & 1230 & 1234 & 1236 & 1239 & 1234 & 1245 & 1258 & 1267 & 1279 \\
\hline Shops and cafes & KMIO & 484 & 478 & 476 & 473 & 469 & 466 & 462 & 459 & 457 & 456 & 455 \\
\hline Offices & KMIP & 258 & 261 & 269 & 273 & 279 & 284 & 287 & 296 & 304 & 311 & 320 \\
\hline Other & KMIQ & 477 & 484 & 485 & 487 & 488 & 490 & 485 & 490 & 497 & 500 & 504 \\
\hline On-licensed premises & KMIR & 60 & 61 & 61 & 60 & 60 & 60 & 66 & 65 & 65 & 64 & 63 \\
\hline Entertainment and recreational: & KMIS & 80 & 79 & 79 & 80 & 80 & 80 & 78 & 79 & 81 & 81 & 83 \\
\hline Theatres and music-halls & KMIU & 1 & 1 & 1 & 1 & 1 & 1 & 1 & 1 & 1 & 1 & 1 \\
\hline Other & KMIV & 79 & 76 & 76 & 77 & 77 & 78 & 76 & 78 & 80 & 80 & 81 \\
\hline Public utility & KMIW & 9 & 8 & 8 & 8 & 8 & 8 & 8 & 8 & 8 & 8 & 7 \\
\hline Educational and cultural & KMIX & 41 & 41 & 42 & 42 & 42 & 43 & 45 & 45 & 45 & 45 & 45 \\
\hline Miscellaneous & KMIY & 61 & 67 & 70 & 70 & 72 & 74 & 74 & 77 & 80 & 81 & 83 \\
\hline Industrial & KMIZ & 250 & 250 & 251 & 250 & 250 & 250 & 252 & 251 & 252 & 249 & 246 \\
\hline Total & KMIH & 1720 & 1729 & 1740 & 1745 & 1749 & 1754 & 1756 & 1771 & 1788 & 1796 & 1806 \\
\hline \multicolumn{13}{|l|}{ Value of assessments ( $£$ million) } \\
\hline Commercial & KMHG & 19652 & 26320 & 27255 & 27622 & 27713 & 27878 & 33013 & 33548 & 33566 & 33427 & 33728 \\
\hline Shops and cafes & KMHH & 5840 & 6801 & 6972 & 6953 & 6863 & 6845 & 8257 & 8311 & 8289 & 8251 & 8321 \\
\hline Offices & KMHI & 5575 & 8625 & 9191 & 9388 & 9555 & 9591 & 10840 & 11034 & 10904 & 10724 & 10794 \\
\hline Other & KMHJ & 8237 & 10894 & 11092 & 11281 & 11295 & 11441 & 13916 & 14203 & 14373 & 14452 & 14613 \\
\hline On-licensed premises & KMHK & 997 & 1311 & 1347 & 1345 & 1334 & 1320 & 1667 & 1652 & 1615 & 1589 & 1567 \\
\hline Entertainment and recreational & KMHL & 1045 & 1310 & 1369 & 1430 & 1416 & 1362 & 1467 & 1483 & 1481 & 1478 & 1466 \\
\hline Cinemas & KMHM & 45 & 79 & 92 & 104 & 106 & 96 & 117 & 115 & 110 & 101 & 102 \\
\hline Theatres and music-halls & KMHN & 20 & 24 & 25 & 26 & 26 & 26 & 34 & 35 & 35 & 35 & 36 \\
\hline Other & КMHO & 980 & 1207 & 1252 & 1300 & 1284 & 1240 & 1316 & 1333 & 1337 & 1342 & 1328 \\
\hline Public utility & KMHP & 3361 & 3828 & 3411 & 3460 & 3444 & 3410 & 3680 & 3668 & 3668 & 3656 & 3471 \\
\hline Educational and cultural & KMHQ & 1672 & 1829 & 1872 & 1902 & 1895 & 1904 & 2359 & 2411 & 2407 & 2397 & 2417 \\
\hline Miscellaneous & KMHR & 1439 & 2142 & 2172 & 2220 & 2218 & 2022 & 2582 & 2646 & 2687 & 2694 & 2697 \\
\hline Industrial & KMHS & 5463 & 6249 & 6202 & 6157 & 6034 & 5935 & 6651 & 6575 & 6453 & 6314 & 6122 \\
\hline Total & KMHA & 33649 & 42985 & 43626 & 44136 & 44053 & 43831 & 51419 & 51983 & 51878 & 51555 & 51468 \\
\hline
\end{tabular}

1 See chapter text.

Source: HM Revenue \& Customs: 02071472941 


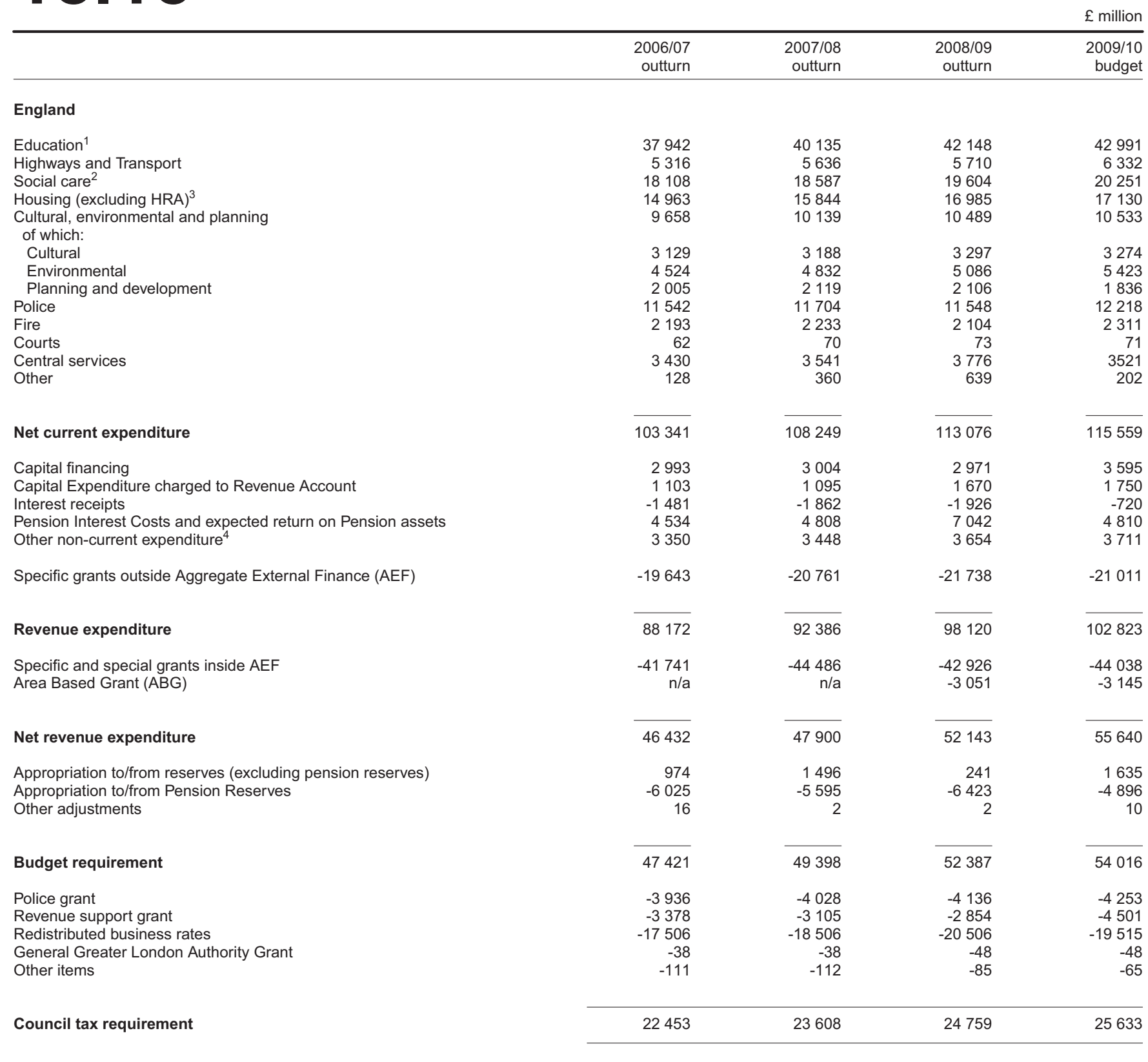

Scotland 


\begin{tabular}{|c|c|c|c|c|}
\hline & $\begin{array}{r}2006 / 07 \\
\text { outturn }\end{array}$ & $\begin{array}{r}2007 / 08 \\
\text { outturn }\end{array}$ & $\begin{array}{r}2008 / 09 \\
\text { outturn }\end{array}$ & $\begin{array}{r}2009 / 10 \\
\text { Budget }\end{array}$ \\
\hline \multicolumn{5}{|l|}{ Wales $^{5}$} \\
\hline Education & 2213.0 & 2325.6 & 2427.5 & 2483.5 \\
\hline Personal social services & 1253.6 & 1302.9 & 1348.8 & 1370.9 \\
\hline Housing 6 & 716.7 & 759.4 & 878.2 & 841.3 \\
\hline Local environmental services ${ }^{7}$ & 353.9 & 356.4 & 393.0 & 410.0 \\
\hline Roads and transport & 283.3 & 296.4 & 329.8 & 316.6 \\
\hline Libraries, culture, heritage, sport and recreation & 259.9 & 267.4 & 285.8 & 270.3 \\
\hline Planning, economic development and community development & 115.2 & 113.6 & 164.7 & 116.5 \\
\hline Council tax benefit and administration ${ }^{8}$ & 32.1 & 31.7 & 31.5 & 30.8 \\
\hline Debt financing costs: counties & 278.0 & 289.0 & 320.5 & 318.9 \\
\hline Central administrative and other revenue expenditure: counties ${ }^{9}$ & 207.4 & 217.3 & 198.0 & 309.1 \\
\hline Total county and county borough council expenditure & 5713.1 & 5959.8 & 6377.8 & 6467.8 \\
\hline Total police expenditure & 601.4 & 623.3 & 642.6 & 675.3 \\
\hline Total national park expenditure & 15.8 & 17.8 & 18.0 & 16.1 \\
\hline Gross revenue expenditure & 6472.4 & 6739.1 & 7183.3 & 7305.0 \\
\hline \multirow{2}{*}{$\begin{array}{l}\text { less specific and special government grants } \\
\text { (except council tax benefit grant) }\end{array}$} & & & & \\
\hline & -1529.7 & -1630.2 & -1809.0 & -1682.3 \\
\hline Net revenue expenditure & 4942.7 & 5108.9 & 5374.3 & 5622.7 \\
\hline Putting to $(+) /$ drawing from $(-)$ reserves & 24.6 & 97.0 & 14.0 & -63.2 \\
\hline Budget requirement & 4967.3 & 5205.9 & 5388.4 & 5559.6 \\
\hline Plus discretionary non-domestic rate relief & 2.6 & 2.5 & 2.4 & 2.6 \\
\hline less revenue support grant & -2951.8 & -3061.6 & -3104.6 & -3191.7 \\
\hline less police grant & -217.0 & -225.0 & -230.5 & -236.3 \\
\hline less re-distributed non-domestic rates income & -730.0 & -791.0 & -868.0 & -894.0 \\
\hline Paid by council tax benefit grant from the Department for Work and Pensions & 177.2 & 184.6 & 194.8 & 203.5 \\
\hline Paid directly by council tax payers & 894.0 & 946.2 & 993.1 & 1036.7 \\
\hline
\end{tabular}

1 Includes mandatory student awards and inter-authority education recoupment.

2 Includes supported employment.

3 Includes mandatory rent allowances and rent rebates.

4 Includes:

(i) Gross expenditure on council tax benefit.

(ii) Expenditure on council tax reduction scheme.

(iii) Discretionary (non-domestic) rate relief.

(iv) Flood defence payments to the Environment Agency.

(v) Bad debt provision.

5 Service expenditure is shown excluding that financed by sales, fees and charges, but including that financed by specific and special government grants.

6 Includes housing benefit and private sector costs such as provision for the homeless. Includes rent rebates granted to HRA tenants which is $100 \%$ grant funded. Excludes council owned housing.

Includes cemeteries and crematoria, community safety, environmental health, consumer protection, waste collection/disposal and central services to the public such as birth registration and elections.

8 Excludes council tax benefit expednditure funded by the specific grant from the Department for Work and Pensions.

9 Includes agricultural services, coastal and flood defence and community councils. Also includes central administrative costs of corporate management, democratic representation and certain costs, such as those relating to backyear or additional pension contributions which should not be allocated to individual services, capital expenditure charged to the revenue account and is net of any interest expected to accrue on balances.

Sources: Communities and Local Government: 02079444158 Scottish Government, Statistical Support for Local Government: 0131245 


\begin{tabular}{|c|c|c|c|c|c|c|c|c|c|c|c|c|}
\hline & & $\begin{array}{r}1999 \\
100\end{array}$ & $\begin{array}{r}2000 \\
/ 01\end{array}$ & $\begin{array}{r}2001 \\
/ 02\end{array}$ & $\begin{array}{r}2002 \\
103\end{array}$ & $\begin{array}{r}2003 \\
/ 04\end{array}$ & $\begin{array}{r}2004 \\
105\end{array}$ & $\begin{array}{r}2005 \\
106\end{array}$ & $\begin{array}{r}2006 \\
107\end{array}$ & $\begin{array}{r}2007 \\
108\end{array}$ & $\begin{array}{c}2008^{1} \\
109\end{array}$ & $\begin{array}{r}2009 \\
/ 10\end{array}$ \\
\hline \multicolumn{13}{|l|}{ England $^{2}$} \\
\hline \multicolumn{13}{|l|}{ Revenue expenditure ${ }^{3}$} \\
\hline Cash £m & KRTN & 53651 & 57329 & 61952 & 65898 & 75244 & 79303 & 84422 & 88172 & 92386 & 98120 & 102823 \\
\hline \multicolumn{13}{|l|}{ Government grants } \\
\hline Cash £m & KRTO & 26421 & 27809 & 31469 & 32634 & 41777 & 45258 & 45838 & 49093 & 51656 & 53015 & 55985 \\
\hline \multicolumn{13}{|l|}{ Redistributed business rates ${ }^{4}$} \\
\hline Cash £m & KRTQ & 13619 & 15407 & 15144 & 16639 & 15611 & 15004 & 18004 & 17506 & 18506 & 20506 & 19515 \\
\hline Percentage of revenue expenditure & KRTR & 25 & 27 & 24 & 25 & 21 & 19 & 21 & 20 & 20 & 21 & 19 \\
\hline \multicolumn{13}{|l|}{ Council tax } \\
\hline Cash £m & KRTS & 13278 & 14200 & 15246 & 16648 & 18946 & 20299 & 21315 & 22453 & 23608 & 24759 & 25633 \\
\hline Percentage of revenue expenditure & KRTT & 25 & 25 & 25 & 25 & 25 & 26 & 25 & 25 & 26 & 25 & 25 \\
\hline Gross revenue expenditure ${ }^{5}$ & $\mathrm{ZBXH}$ & 3424 & 3605 & 4350 & 4709 & 5243 & 5786 & 6128 & 6472 & 6739 & 7184 & 7305 \\
\hline General government grants ${ }^{6}$ & $\mathrm{ZBXI}$ & 2093 & 2234 & 2345 & 2541 & 2743 & 2817 & 2987 & 3169 & 3287 & 3335 & 3428 \\
\hline Specific government grants ${ }^{7}$ & ZBXG & 80 & 94 & 601 & 779 & 1005 & 1381 & 1473 & 1530 & 1630 & 1809 & 1682 \\
\hline \multicolumn{13}{|l|}{ Share of redistributed } \\
\hline business rates & ZBXJ & 656 & 638 & 697 & 643 & 660 & 672 & 672 & 730 & 791 & 868 & 864 \\
\hline Council tax income ${ }^{8}$ & $\mathrm{ZBXK}$ & 596 & 670 & 716 & 776 & 861 & 924 & 1012 & 1071 & 1131 & 1188 & 1240 \\
\hline Other $^{9}$ & $\mathrm{ZBXL}$ & -1 & -31 & -10 & -30 & -25 & -8 & -16 & -27 & -99 & -16 & 61 \\
\hline
\end{tabular}

1 Budget estimates.

2 Produced on a non-Financial Reporting Standard 17 (FRS17) basis.

6 Includes all unhypothecated grants, namely revenue support grant, police

3 The sum of government grants, business rates and local taxes does not

normally equal revenue expenditure because of the use of reserves.

grant, council tax reduction scheme grant, transitional grant and the adjustment

1993-94 to 2003-04 includes City of London Offset.

7 Comprises specific and supplementary grants, excluding police grant.

5 Gross revenue expenditure is total local authority expenditure on services, plus capital charges, but net of any income from sales, fees, and charges and other non-grant sources. It includes expenditure funded by specific grants. The figures have been adjusted to account for FRS17 pension costs.

8 This includes community council precepts, and income covered by charge/council tax benefit grant, but excludes council tax reduction scheme.

9 This includes use of, or contributors to, local authority reserves and other minor adjustments.

Sources: Communities and Local Government: 02079444158 , Welsh Assembly Government: 02920825355

\subsection{7}

$£$ million

\begin{tabular}{|c|c|c|c|c|c|c|}
\hline & $\begin{array}{r}\text { Central government } \\
\text { grants }^{1}\end{array}$ & $\begin{array}{l}\text { Other grants and } \\
\text { contributions }^{2}\end{array}$ & $\begin{array}{l}\text { Use of usable } \\
\text { capital receipts }\end{array}$ & $\begin{array}{r}\text { BCA/SCE(R)Single } \\
\text { Capital Pot }\end{array}$ & $\begin{array}{r}\text { BCA/SCE }(\mathrm{R}) \text { Separate } \\
\text { Programme Element }\end{array}$ & $\begin{array}{r}\text { Other borrowing and } \\
\text { credit arrangements } \\
\text { not supported by } \\
\text { central government }\end{array}$ \\
\hline \multicolumn{7}{|c|}{ Financial year } \\
\hline & KRVM & $14 \mathrm{~V} 9$ & I4VA & I4VB & $14 \mathrm{VC}$ & I4VD \\
\hline 1999/00 & 1161 & 571 & 1599 & 1051 & 1250 & \\
\hline $2000 / 01$ & 1298 & 762 & 1592 & 2271 & 945 & \\
\hline 2001/02 & 2027 & 757 & 1975 & 1173 & 1378 & \\
\hline $2003 / 04$ & 2642 & 869 & 1988 & 2583 & 1326 & \\
\hline $2004 / 05$ & 3196 & 1080 & 2647 & 2959 & 704 & 1061 \\
\hline $2005 / 06$ & 3909 & 1377 & 2812 & 2932 & 947 & 2251 \\
\hline $2006 / 07$ & 4083 & 1344 & 2628 & 2734 & 630 & 2291 \\
\hline $2007 / 08$ & 7007 & 2019 & 2665 & 2296 & 630 & 3186 \\
\hline 2008/09 & 5733 & 1978 & 2040 & 2257 & 760 & 4224 \\
\hline
\end{tabular}

\begin{tabular}{|c|c|c|c|c|c|}
\hline & \multirow[b]{2}{*}{ Use of other resources ${ }^{3}$} & \multicolumn{4}{|c|}{ Revenue financing of capital expenditure, of which: } \\
\hline & & Housing revenue account & Major repairs reserve & General Fund & Total resources used \\
\hline \multicolumn{6}{|c|}{ Financial year } \\
\hline & I4VE & I4VF & I4VG & $14 \mathrm{VH}$ & $14 \mathrm{VI}$ \\
\hline 1999/00 & 231 & 327 & .. & 808 & 6998 \\
\hline $2000 / 01$ & 304 & 218 & .. & 896 & 8288 \\
\hline $2001 / 02$ & 387 & 1505 &.. & 825 & 10028 \\
\hline $2003 / 04$ & 262 & 212 & 1388 & 1055 & 12326 \\
\hline $2004 / 05$ & .. & 187 & 1440 & 1130 & 14404 \\
\hline $2005 / 06$ & .. & 238 & 1327 & 1004 & 16797 \\
\hline $2006 / 07$ & .. & 240 & 1337 & 1185 & 16472 \\
\hline $2007 / 08$ & .. & 208 & 1180 & 1204 & 20395 \\
\hline 2008/09 & .. & 228 & 1224 & 1789 & 20233 \\
\hline
\end{tabular}

1 2007-08 includes an exceptional item, £1.7 billion grant from DfT to GLA 3 Use of monies set aside as provision for credit liabilities to finance capital ex( $\mathrm{TfL}$ ) for the $£ 1.7$ billion payment to Metronet.

Source: Department for Communities and Local Government: 03034442121

Public Bodies, National Lottery and European Structural Fund. 


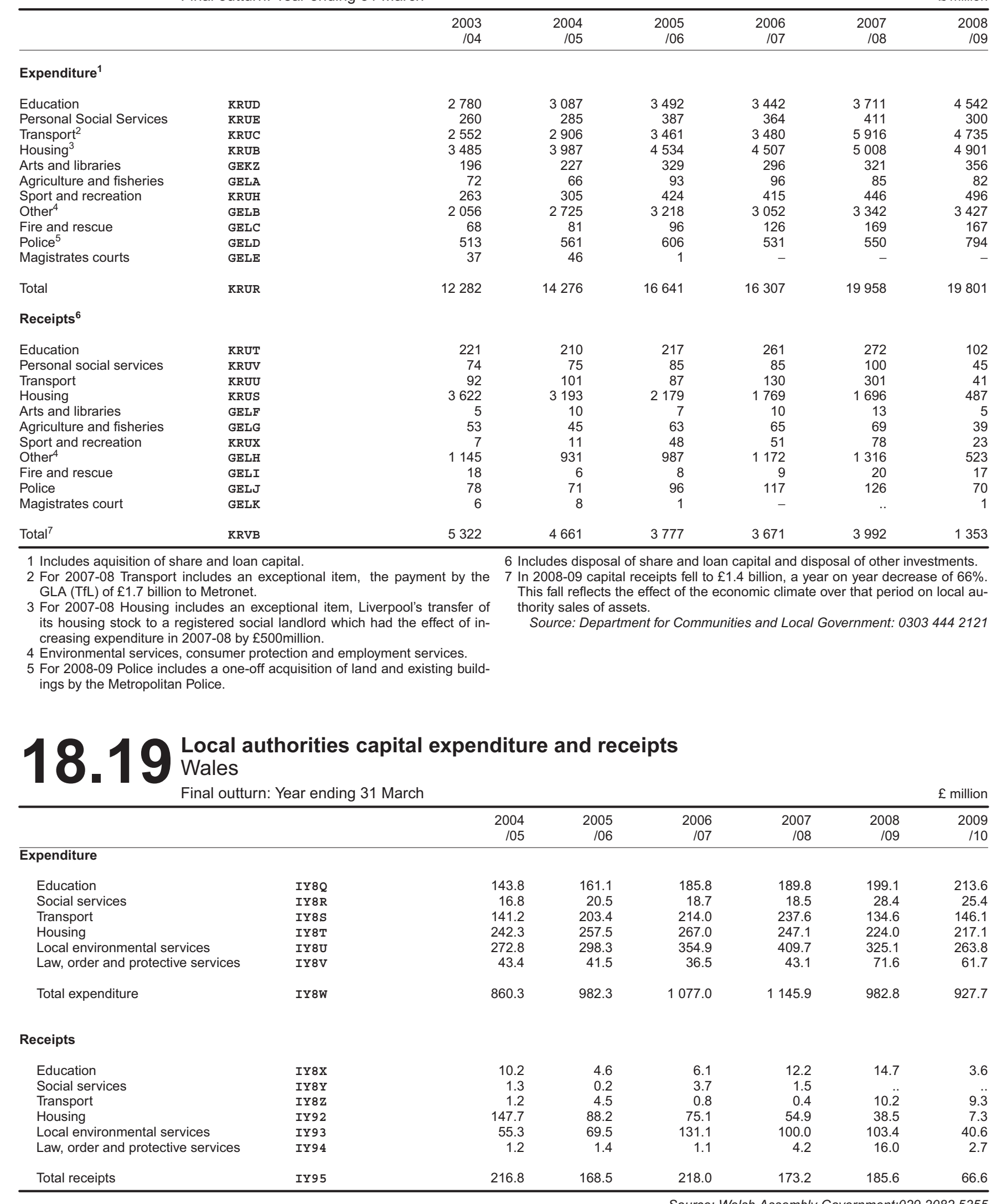




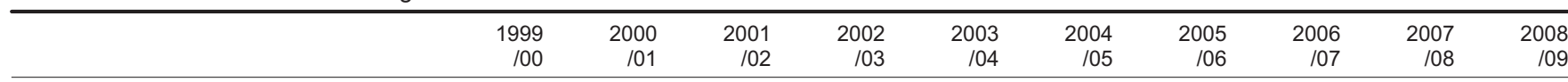

Out of revenue: ${ }^{1}$ Total

General Fund Services: Education

Libraries, museums and galleries

Social work

protective services

Roads and Transport ${ }^{2}$

Environmental services

Planning

Leisure and recreation

Other services

Other general fund expenditure ${ }^{3}$

Housing

Trading services:

Passenger transport

Ferries

Harbours, docks and piers

Road bridges

Slaughterhouses

Markets

Other trading services

Loan charges: ${ }^{4}$ Total

Allocated to :

General Fund services

Housing

Trading services

On capital works: ${ }^{4}$ Total

General Fund Services:

Education

Libraries, museums and galleries

Social work

Law, order and

protective services

Roads and Transport

Environmental services

Planning

Leisure and recreation

Administrative buildings and

equipment

Other services

Housing

Trading Services:

Ferries

Harbours, docks and piers

Airports

Shipping, Airports, Transport piers $06 \mathrm{X}$

\& Ferry Terminals

Road bridges

Slaughterhouses

Other trading services

KQTA
Law, order and

KQTB $74296267884168 \quad 84282179290268101396791096459812021453121430561265358513255975$

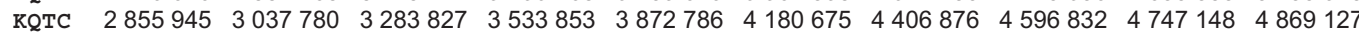

$\begin{array}{llllllllllll}\text { KOTD } & 131696 & 134174 & 138318 & 152308 & 160540 & 161650 & 168953 & 164976 & 163185 & 170177\end{array}$ KQTE $1519191 \quad 16328431793732 \quad 2173752 \quad 2400652 \quad 262113428080402994486 \quad 3192214 \quad 3408851$

KQTF $100600010470341088791 \quad 1130693 \quad 1226067 \quad 13060851501854146964415064321631037$ $\begin{array}{llllllllllll}\text { KQTG } & 527018 & 564738 & 506326 & 601454 & 611721 & 635329 & 673167 & 625341 & 633828 & 678922\end{array}$ $\begin{array}{llllllllllll}\text { KQTH } & 373050 & 393333 & 414975 & 484177 & 525556 & 581220 & 635475 & 670308 & 708736 & 757555\end{array}$ $\begin{array}{lllllllllll}\text { KQTH } & 373050 & 393333 & 414975 & 484177 & 525556 & 581220 & 635475 & 670308 & 708736 & 757555 \\ \text { KQTI } & 198285 & 194771 & 223414 & 265315 & 282572 & 299182 & 351617 & 366803 & 372445 & 437261\end{array}$ $\begin{array}{llllllllllll}\text { KQTJ } & 375579 & 387115 & 401904 & 426495 & 472120 & 494237 & 520612 & 543047 & 547132 & 572111\end{array}$ $\begin{array}{llllllllllll}\text { KQTL } & 435155 & 465612 & 572136 & 515661 & 585425 & 681288 & 948167 & 702554 & 782035 & 730934\end{array}$ $\begin{array}{llllllllll}\text { KQTM } & 7707 & 26768 & 4794 & 6560 & 2240 & 3798 & 6692 & 9065 & 430\end{array}$ KQTN $18213801886189195444422242092295005 \quad 2459146 \quad 26092282740592 \quad 27885372976629$

\begin{tabular}{|c|c|c|c|c|c|c|c|c|c|c|}
\hline KQTO & 87321 & 80355 & 61899 & 74062 & 92782 & 106445 & 103461 & 102336 & 100104 & 110042 \\
\hline KQTR & 336 & 162 & 343 & 427 & 441 & 282 & 353 & 355 & 315 & \\
\hline KQTS & 9709 & 10005 & 9650 & 11493 & 11768 & 13759 & 14308 & 18483 & 21907 & 238 \\
\hline KQTT & 15923 & 13604 & 10912 & 12222 & 13405 & 12407 & 11995 & 8495 & 8312 & \\
\hline KQTV & 8231 & 8606 & 6914 & 7267 & 11235 & 13276 & 12366 & 16279 & 22005 & 17 \\
\hline KQTW & 4 & & & & & & & & & \\
\hline KQTX & 14106 & 23844 & 16657 & 17995 & 14824 & 15353 & 17447 & 16793 & 18461 & \\
\hline KQTY & 39012 & 24134 & 17423 & 24658 & 41109 & 51368 & 46992 & 41931 & 29104 & \\
\hline KQTZ & 1109379 & 1100690 & 1114161 & 1269994 & 1131368 & 997678 & 1012287 & 1000767 & 1036023 & 1060 \\
\hline KMHV & 701515 & 708822 & 739351 & 738870 & 772852 & 772648 & 792404 & 782002 & 806806 & $854 \mathrm{~s}$ \\
\hline KMHW & 402936 & 386512 & 369943 & 525201 & 348180 & 212440 & 210856 & 214395 & 201297 & 1977 \\
\hline KMHX & 4928 & 5356 & 4867 & 5923 & 10336 & 12590 & 9027 & 4370 & 27920 & \\
\hline KQUA & 816473 & 802672 & 929631 & 972049 & 1052310 & 1264031 & 1572281 & 1952249 & 2182509 & 2554 \\
\hline KQUB & 557119 & 538843 & 610485 & 662869 & 767122 & 1006150 & 1160818 & 1462620 & 1652425 & 1850 \\
\hline KQUC & 136508 & 127781 & 143268 & 157439 & 172227 & 199387 & 310054 & 402865 & 464827 & 4792 \\
\hline KQUD & 10261 & 5834 & 8683 & 19018 & 12043 & 24796 & 22762 & 24210 & 29963 & 39 \\
\hline KQUE & 22097 & 21539 & 31359 & 30116 & 31966 & 33450 & 37877 & 50327 & 65449 & 63 \\
\hline KQUF & 37132 & 35761 & 39901 & 53268 & 65477 & 65154 & 51146 & 60287 & 68680 & 1010 \\
\hline KQUG & 108500 & 117485 & 147975 & 147357 & 200278 & 258071 & 308366 & 418987 & 484669 & 479 \\
\hline KQUH & 14936 & 17944 & 16396 & 17957 & 20567 & 40773 & 55020 & 43104 & 101325 & 121 \\
\hline KQUI & 52045 & 47684 & 33312 & 40241 & 36496 & 61544 & 76043 & 66063 & 121596 & 1240 \\
\hline KQUJ & 52365 & 44516 & 39240 & 50558 & 71486 & 74116 & 83681 & 98275 & 136029 & 167 \\
\hline KQUK & 35824 & 34633 & 53189 & 68438 & 48896 & 64414 & 84569 & 113896 & & \\
\hline KQUL & 87451 & 85666 & 97162 & 78477 & 107686 & 184445 & 131300 & 184606 & 179887 & 2749 \\
\hline KQUM & 255019 & 255189 & 300054 & 284418 & 261715 & 241107 & 382697 & 454838 & 507905 & 68065 \\
\hline KQUN & 4335 & 8640 & 19092 & 24762 & 23473 & 16774 & 28766 & 34791 & 22179 & 227 \\
\hline KQUR & 1030 & 23 & 467 & 1 & 111 & 608 & 195 & 547 & . & \\
\hline KQUS & 1389 & 6192 & 15898 & 20361 & 19503 & 12024 & 12899 & 5855 & .. & \\
\hline KQUT & .. & 607 & 663 & 1031 & 609 & 572 & 663 & 798 & & \\
\hline J96x & .. & .. & .. & .. & .. & .. & .. & .. & 18654 & 1401 \\
\hline KQUU & 600 & 964 & 882 & 2386 & 2395 & 442 & 12106 & 22865 & - & \\
\hline KQUV & 12 & & 40 & 116 & 82 & - & - & - & - & \\
\hline КМHY & 1304 & 854 & 1142 & 867 & 773 & 3128 & 2903 & 4726 & 3525 & 87 \\
\hline
\end{tabular}

Source: Scottish Government, Statistical Support for Local Government: 0131

1 Gross expenditure less inter-authority and inter-account transfers.

2 Including general fund support for transport (LA and NON-LA).

3 General fund contributions to Housing and Trading services (excluding

transport), are also included in the expenditure figures for these services.

4 Expenditure out of loans, government grants and other capital receipts. 


\begin{tabular}{|c|c|c|c|c|c|c|c|c|c|c|c|c|}
\hline & & $\begin{array}{r}1998 \\
199\end{array}$ & $\begin{array}{r}1999 \\
100\end{array}$ & $\begin{array}{r}2000 \\
/ 01\end{array}$ & $\begin{array}{r}2001 \\
/ 02\end{array}$ & $\begin{array}{r}2002 \\
103\end{array}$ & $\begin{array}{r}2003 \\
104\end{array}$ & $\begin{array}{r}2004 \\
105\end{array}$ & $\begin{array}{r}2005 \\
106\end{array}$ & $\begin{array}{r}2006 \\
107\end{array}$ & $\begin{array}{r}2007 \\
108\end{array}$ & $\begin{array}{r}2008 \\
109\end{array}$ \\
\hline \multicolumn{13}{|l|}{ Revenue account } \\
\hline Non-Domestic Rates ${ }^{1}$ & KQXA & 1437646 & 1440522 & 1662691 & 1553926 & 1718104 & 1804423 & 1895941 & 1897073 & 1883769 & 1859727 & 1962800 \\
\hline Council tax & KPUC & 146366 & 1193693 & 1273316 & 1363399 & 1459212 & 1532071 & 1614808 & 1720305 & 1811577 & 1889913 & 1908972 \\
\hline \multicolumn{13}{|l|}{ Government grants } \\
\hline $\begin{array}{l}\text { General Revenue Funding }{ }^{2} \\
\text { Council tax }\end{array}$ & $\mathrm{KQXC}$ & 3483815 & 3537043 & 3440842 & 3935328 & 4557867 & 5037140 & 5266054 & 5567902 & 5777204 & 6169645 & 7425884 \\
\hline rebate grants & KPUD & 274940 & 275789 & 279459 & 285131 & 293606 & 307733 & 344899 & 354067 & 359159 & 354030 & 351165 \\
\hline \multicolumn{13}{|l|}{ Other grants and } \\
\hline subsidies & $\mathrm{KQXI}$ & 1642045 & 1778216 & 1891839 & 2061297 & 2141543 & 2479311 & 2823820 & 2940137 & 3147497 & 3310712 & 2602219 \\
\hline Sales & $\mathrm{KQXJ}$ & 39595 & 43660 & 49826 & & & & & & & & \\
\hline Fees and charges ${ }^{3}$ & $\mathrm{KQXK}$ & 1668223 & 1682385 & 1776455 & 1789428 & 1954337 & 1785672 & 1845161 & 1951315 & 2039217 & 2125114 & 2253653 \\
\hline Other income & KQXL & 324932 & 398894 & 453458 & 490574 & 712423 & 515897 & 709226 & 1003925 & 961693 & 875369 & 766126 \\
\hline \multicolumn{13}{|l|}{ Capital account } \\
\hline Sale of fixed assets & KQXM & 335037 & 303582 & 149504 & 165016 & 207388 & 222844 & 355069 & 366302 & 451353 & 513913 & 229805 \\
\hline Revenue contributions & סצחצי סצי & 204982 & 213564 & 10912 & 147760 & 230778 & 212533 & 210503 & 693 & 109740 & 173668 & 196 \\
\hline Transfer from special & KQXP & 204982 & 213564 & 210912 & $14 / 760$ & 239718 & 212533 & & & & & 1968 \\
\hline Other receipts ${ }^{4}$ & KMGV & 45028 & 39014 & 45351 & 90360 & 75846 & 114745 & 130575 & 261872 & 595722 & 826145 & 74223 \\
\hline
\end{tabular}

1 This is the Distributable Amount of Non-Domestic Rates.

3 From 2001-02 onwards, fees \& charges incorporates sales.

2 Revenue Support Grant re-named General Revenue Funding from 4 Figures include public sector contributions from 2001-02 onwards. 2008-09.

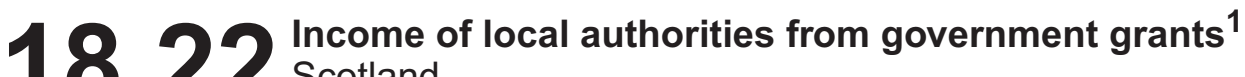 \\ 18.22 soctand \\ Year ending 31 March}

$£$ thousand

\begin{tabular}{|c|c|c|c|c|c|c|c|c|c|c|c|}
\hline & & $\begin{array}{r}1999 \\
/ 00\end{array}$ & $\begin{array}{r}2000 \\
/ 01\end{array}$ & $\begin{array}{r}2001 \\
102\end{array}$ & $\begin{array}{r}2002 \\
103\end{array}$ & $\begin{array}{r}2003 \\
104\end{array}$ & $\begin{array}{r}2004 \\
105\end{array}$ & $\begin{array}{r}2005 \\
106\end{array}$ & $\begin{array}{r}2006 \\
107\end{array}$ & $\begin{array}{r}2007 \\
108\end{array}$ & $\begin{array}{r}2008 \\
109\end{array}$ \\
\hline General fund services & KQYA & 818537 & 935452 & 1032591 & 952692 & 1029338 & 1207912 & 1358190 & 1524829 & 1503002 & 1041117 \\
\hline Education & KQYB & 225668 & 324340 & 380726 & 251333 & 217743 & 287226 & 327905 & 439678 & 418636 & 96477 \\
\hline $\begin{array}{l}\text { Libraries, museums and } \\
\text { galleries }\end{array}$ & KQYC & 507 & 634 & 1137 & 5359 & 1517 & 763 & 818 & 1394 & 1523 & 1869 \\
\hline Social work & KQYD & 71611 & 78611 & 86533 & 114591 & 205229 & 240665 & 236774 & 222551 & 222741 & 122999 \\
\hline Law, order and & & & & & & & & & & & \\
\hline protective services & KQYE & 382246 & 401485 & 423636 & 445275 & 476681 & 512501 & 597322 & 601593 & 569637 & 594770 \\
\hline $\begin{array}{l}\text { Environmental services } \\
\text { Planning and }\end{array}$ & KQYG & 71 & 301 & 2272 & 5407 & 18120 & 39971 & 45338 & 55173 & 59112 & 7219 \\
\hline Economic Development & $\mathrm{KQYH}$ & 4311 & 4375 & 20351 & 19434 & 21517 & 20767 & 31293 & 33750 & 41068 & 97250 \\
\hline Leisure and recreation & KQYI & 1491 & 2377 & 3322 & 2968 & 3732 & 5830 & 6256 & 9194 & 12796 & 15532 \\
\hline Other services & KQYK & 64203 & 65627 & 64714 & 50661 & 57519 & 65151 & 80780 & 112201 & 114690 & 63228 \\
\hline Housing & KQYL & 959276 & 956239 & 1028529 & 1188626 & 1449616 & 1614976 & 1580504 & 1622049 & 1805354 & 1560883 \\
\hline Trading services & KQYM & 403 & 148 & 177 & 225 & 357 & 932 & 1443 & 619 & 2356 & 219 \\
\hline Total & KMGZ & 5315259 & 5332681 & 5996625 & 6699410 & 7516451 & 8089874 & 8508039 & 8924701 & 9480357 & 10028103 \\
\hline
\end{tabular}

1 Including grants for capital works.

Source: Scottish Government, Statistical Support for Local Government: 0131

2 Decrease in general fund services in 2008-09 is due to the rolling-up of

ring-fenced grants into General Revenue Funding.

3 General revenue funding. 
Expenditure of local authorities

Northern Ireland

Years ending 31 March

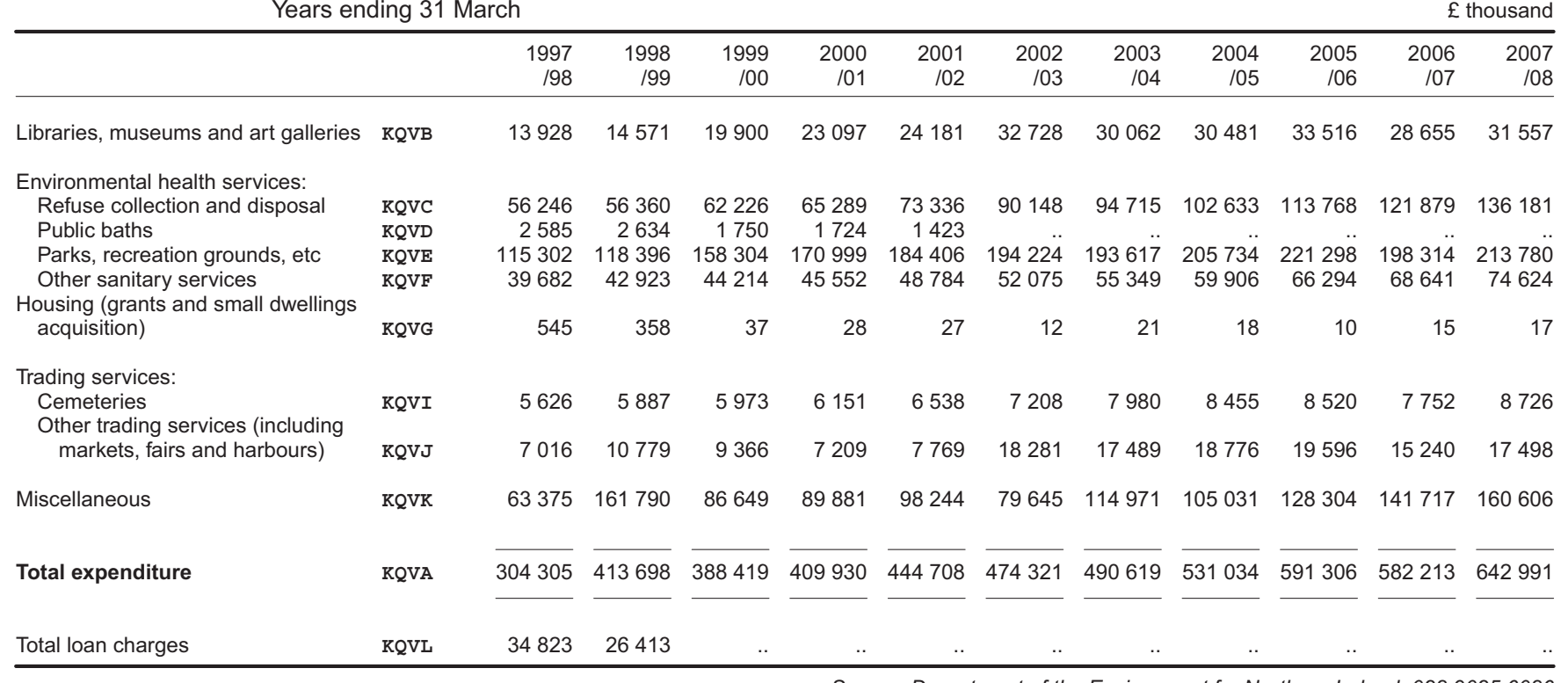

Source: Department of the Environment for Northern Ireland: 02890256086 